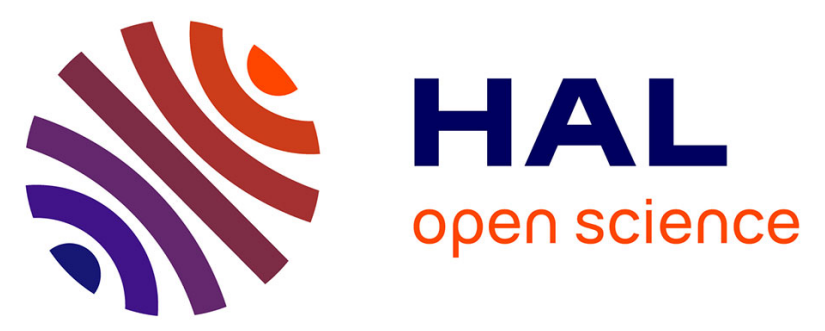

\title{
Rotavirus genotypes co-circulating in Europe between 2006 and 2009 as determined by EuroRotaNet, a pan-European collaborative strain surveillance network
}

M. Iturriza-Gómara, T. Dallman, K. Bányai, B. Böttiger, J. Buesa, S.

Diedrich, L. Fiore, K. Johansen, M. Koopmans, N. Korsun, et al.

\section{To cite this version:}

M. Iturriza-Gómara, T. Dallman, K. Bányai, B. Böttiger, J. Buesa, et al.. Rotavirus genotypes co-circulating in Europe between 2006 and 2009 as determined by EuroRotaNet, a pan-European collaborative strain surveillance network. Epidemiology and Infection, 2011, 139 (6), pp.895-909. 10.1017/S0950268810001810 . hal-02291017

\section{HAL Id: hal-02291017 https://u-bourgogne.hal.science/hal-02291017}

Submitted on 18 Sep 2019

HAL is a multi-disciplinary open access archive for the deposit and dissemination of scientific research documents, whether they are published or not. The documents may come from teaching and research institutions in France or abroad, or from public or private research centers.
L'archive ouverte pluridisciplinaire HAL, est destinée au dépôt et à la diffusion de documents scientifiques de niveau recherche, publiés ou non, émanant des établissements d'enseignement et de recherche français ou étrangers, des laboratoires publics ou privés. 


\title{
Rotavirus genotypes co-circulating in Europe between 2006 and 2009 as determined by EuroRotaNet, a pan-European collaborative strain surveillance network
}

\author{
M. ITURRIZA-GÓMARA ${ }^{1 *}$, T. DALLMAN², K. BÁNYAI ${ }^{3}$, B. BÖTTIGER ${ }^{4}$, \\ J. BUESA ${ }^{5}$, S. DIEDRICH ${ }^{6}$, L. FIORE ${ }^{7}$, K. JOHANSEN ${ }^{8}$, M. KOOPMANS $^{11}$, \\ N. KORSUN ${ }^{10}$, D. KOUKOU ${ }^{9}$,A. KRONEMAN ${ }^{11}$, B. LÁSZLÓ ${ }^{12}$, M. LAPPALAINEN ${ }^{13}$, \\ L. MAUNULA ${ }^{14}$, A. MAS MARQUES ${ }^{6}$, J. MATTHIJNSSENS ${ }^{15}$, S. MIDGLEY ${ }^{4}$, \\ Z. MLADENOVA ${ }^{10}, \mathrm{~S}$. NAWAZ ${ }^{1}$, M. POLJSAK-PRIJATELJ ${ }^{16}$, P. POTHIER $^{17}$, \\ F. M. RUGGERI ${ }^{18}$, A. SANCHEZ-FAUQUIER ${ }^{19}$, A. STEYER ${ }^{16}$, \\ I. SIDARAVICIUTE-IVASKEVICIENE ${ }^{20}$, V. SYRIOPOULOU ${ }^{9}$, A. N. TRAN, \\ V. USONIS ${ }^{20}$, M. VAN RANST ${ }^{15}$, A. DE ROUGEMONT ${ }^{17}$ AND J. GRAY ${ }^{1}$ \\ ${ }^{1}$ Enteric Virus Unit, Virus Reference Department, Centre for Infections, Health Protection Agency (HPA), \\ London, UK, ${ }^{2}$ Bioinformatics Unit: Statistics, Modelling \& Bioinformatics, Centre for Infections, HPA, \\ London, UK, ${ }^{3}$ Veterinary Medical Research Institute, Budapest, Hungary; ${ }^{4}$ Virus Reference Laboratory, \\ Statens Serum Institute, Copenhagen, Denmark; ${ }^{5}$ University of Valencia, Valencia, Spain ; ${ }^{6}$ Molecular Virology, \\ Robert-Koch Institut, Berlin, Germany; ${ }^{7}$ Centro Nazionale per la Ricerca e la Valutazione dei Prodotti \\ Immunobiologici, Istituto Superiore di Sanità, Rome, Italy; ${ }^{8}$ Swedish Institute for Infectious Disease Control, \\ Solna, Sweden; ${ }^{9}$ First Department of Pediatrics, Athens University, Aghia Sophia Children's Hospital, Athens, \\ Greece; ${ }^{10}$ National Reference Laboratory of Enteroviruses, Department of Virology, National Centre for \\ Infectious and Parasitic Diseases, Sofia, Bulgaria; ${ }^{11}$ National Institute for Public Health and the Environment, \\ Bilthoven, The Netherlands; ${ }^{12}$ Department of Medical Microbiology, University of Debrecen, Debrecen, \\ Hungary ; ${ }^{13}$ Laboratory Services (HUSLAB), Helsinki University Hospital, ${ }^{14}$ Department of Food and \\ Environmental Hygiene, University of Helsinki, Finland; ${ }^{15}$ Department of Microbiology and Immunology, Rega \\ Institute for Medical Research, University of Leuven, Belgium; ${ }^{16}$ University of Ljubljana, Faculty of Medicine, \\ Institute of Microbiology and Immunology, Slovenia; ${ }^{17}$ Laboratoire de Virologie, CHI de Bocage, Dijon, France ; \\ ${ }^{18}$ Dipartimento di Sanità Pubblica Veterinaria e Sicurezza Alimentare, Istituto Superiore di Sanità, Rome, Italy, \\ ${ }^{19}$ Centro National de Microbiologia, Instituto de Salud Carlos III, Madrid, Spain; \\ ${ }^{20}$ Vilnius University Centre of Paediatrics, Vilnius, Lithuania
}

(Accepted 30 June 2010)

\section{SUMMARY}

EuroRotaNet, a laboratory network, was established in order to determine the diversity of co-circulating rotavirus strains in Europe over three or more rotavirus seasons from 2006/2007 and currently includes 16 countries. This report highlights the tremendous diversity of rotavirus strains co-circulating in the European population during three years of surveillance since 2006/ 2007 and points to the possible origins of these strains including genetic reassortment and interspecies transmission. Furthermore, the ability of the network to identify strains circulating with an incidence of $\geqslant 1 \%$ allowed the identification of possible emerging strains such as G8 and G12 since the beginning of the study; analysis of recent data indicates their increased incidence. The introduction of universal rotavirus vaccination in at least two of the participating countries, and partial vaccine coverage in some others may provide data on diversity driven by vaccine introduction and possible strain replacement in Europe.

Key words: Rotavirus.

\footnotetext{
* Author for correspondence: Dr M. Iturriza-Gómara, Enteric Virus Unit, Virus Reference Department, Centre for Infections, Health Protection Agency (HPA), London, UK. (Email: miren.iturriza@hpa.org.uk)
} 


\section{INTRODUCTION}

Rotaviruses are a major cause of gastroenteritis in children aged $<5$ years worldwide $[1,2]$ and of acute diarrhoea in the young of many other mammalian species (calves, piglets, lambs, rabbits, etc.). They cause more than 600000 deaths each year, mostly of infants and young children in developing countries [1], and are a significant cause of morbidity and hospitalizations in developed countries.

Rotaviruses are classified according to the serological cross-reactivity of the inner capsid protein VP6 into five groups (A-E) and two more groups $(F, G)$ are likely to exist. Within group A rotaviruses, there are subgroups (I, II, I+II, non-I, non-II) characterized by their reactivities with two VP6-specific monoclonal antibodies [3]. More recently, RT-PCR has been applied to the subgroup classification of human rotaviruses, and only two major subgroups have been identified [4].

In order to characterize strains on the basis of their surface proteins VP4 and VP7, a binary classification scheme distinguishing types has been established for group A rotaviruses. The system differentiates G-types (VP7-specific, G for glycoprotein) and P-types (VP4-specific, $\mathrm{P}$ for protease-sensitive protein). To date, 23 different G-types and 31 P-types have been described [5-9], indicating extensive genomic diversity within group A rotaviruses.

Rotaviruses possess a genome of 11 segments of dsRNA and VP4 and VP7 are coded for by different RNA segments (segments 4 and 7, 8 or 9 depending on strain, respectively). As rotaviruses were found to reassort readily in doubly infected cells in vitro and in vivo [7, 10-12], various combinations of VP4 and VP7 types have been observed in natural rotavirus isolates [13-16].

The European Rotavirus Network (EuroRotaNet), was established in January 2007 [17], and has conducted rotavirus strain surveillance in Europe for three consecutive years. Participation in EuroRotaNet is voluntary, and the network activities are funded between the collaborating institutes and GlaxoSmithKline Biologicals (GSK) and Sanofi Pasteur-MSD (SPMSD). The participating institutes provide expertise, laboratory space, equipment and supervision, and funding for labour and consumable costs associated with strain characterization is provided in the form of an unrestricted collaborative grant in equal parts from GSK and SPMSD, and administered through the Centre for Infections, Health Protection Agency, London, UK.
The study was undertaken in order to gather comprehensive information of the rotavirus types cocirculating throughout Europe, including both urban and rural settings, and encompassing at least three consecutive rotavirus seasons. The aims of the study were (i) to develop methods and algorithms for effective rotavirus typing ( $\mathrm{G}$ and $\mathrm{P})$ and characterization (including VP6 and NSP4 genotypes) and to monitor the effectiveness of current genotyping methods and respond to changes associated with genetic drift and shift; (ii) to describe in detail the molecular epidemiology of rotavirus infections in Europe, during consecutive rotavirus seasons, through genotyping of rotavirus-positive samples collected throughout each country; and (iii) to monitor the emergence and spread of common and novel rotavirus strains within Europe and develop the infrastructure that may serve as a platform for future surveillance activities and nested studies. These studies will be used to monitor the effectiveness of a rotavirus vaccine in the general population, through monitoring the reduction in disease associated with common rotavirus types; to detect the possible vaccine-induced emergence of antibody escape mutants and the possible emergence in the general population of genotypes other than those included in the vaccine and reassortants between vaccine and naturally circulating wild-type strains.

\section{MATERIALS AND METHODS}

\section{Samples}

Hospitals, paediatricians and general medical practitioners that were willing to participate in the study over several years were identified in each of the participating countries in order to allow valid comparisons to be made between each rotavirus season and within each member country. Faecal samples submitted for the routine laboratory investigation of infantile gastroenteritis and positive for group A rotavirus antigen were collected for genotyping. A total of 19140 rotavirus-positive samples were genotyped between September 2006 and August 2009 in 15 European countries (Table 1).

\section{Rotavirus genotyping}

Rotavirus strains were characterized through genotyping using standardized methods to identify their G- and P-types [Manual of rotavirus detection and characterization methods, WHO (http://www.who.int/ nuvi/rotavirus/WHO_IVB_08.17_eng.pdf); European 
Table 1. Number of rotavirus strains in the EuroRotaNet database per country and rotavirus season (includes data for September to August in each of the three season from 2006 to 2009; entries for which date of sample collection was incomplete were excluded)

\begin{tabular}{|c|c|c|c|c|c|c|}
\hline Country & $\begin{array}{l}2006 / \\
2007\end{array}$ & $\begin{array}{l}2007 / \\
2008\end{array}$ & $\begin{array}{l}2008 / \\
2009\end{array}$ & $\begin{array}{l}\text { Study } \\
\text { target }\end{array}$ & Total & $\begin{array}{l}\% \text { target } \\
\text { achieved }\end{array}$ \\
\hline Belgium & & 607 & 214 & 940 & 821 & $87 \cdot 3$ \\
\hline Bulgaria & 338 & 328 & 299 & 940 & 965 & $102 \cdot 7$ \\
\hline Denmark & 185 & 270 & 245 & 1410 & 700 & $49 \cdot 7$ \\
\hline Finland & 142 & 266 & 226 & 1410 & 634 & $45 \cdot 0$ \\
\hline France & 578 & 764 & 620 & 2193 & 1962 & $89 \cdot 5$ \\
\hline Germany & 39 & 964 & 752 & 2877 & 1755 & $61 \cdot 0$ \\
\hline Greece & ns & ns & 96 & 470 & 96 & $20 \cdot 4$ \\
\hline Hungary & 388 & 586 & 436 & 1410 & 1410 & $100 \cdot 0$ \\
\hline Italy & 347 & 1290 & 748 & 2103 & 2385 & $113 \cdot 4$ \\
\hline Lithuania & 1 & 564 & 446 & 940 & 1011 & $107 \cdot 6$ \\
\hline $\begin{array}{l}\text { The } \\
\text { Netherland }\end{array}$ & 16 & 139 & 838 & 1410 & 993 & $70 \cdot 4$ \\
\hline Romania & n.s. & n.s. & t.b.u. & 470 & - & $0 \cdot 0$ \\
\hline Slovenia & 353 & 631 & 234 & 1410 & 1218 & $86 \cdot 4$ \\
\hline Spain & 544 & 662 & 530 & 2352 & 1736 & $73 \cdot 8$ \\
\hline Sweden & 32 & 578 & 115 & 1410 & 725 & $51 \cdot 4$ \\
\hline UK & 844 & 910 & 975 & 2115 & 2729 & $129 \cdot 0$ \\
\hline Total & 3807 & 8559 & 6774 & 23860 & 19140 & $80 \cdot 2$ \\
\hline
\end{tabular}

n.s., No sampling; t.b.u., to be uploaded.

Belgium, Bulgaria and Lithuania joined the network in 2008, Greece and Romania in 2009. Only data uploaded up to 31 December 2009 is included in the analysis.

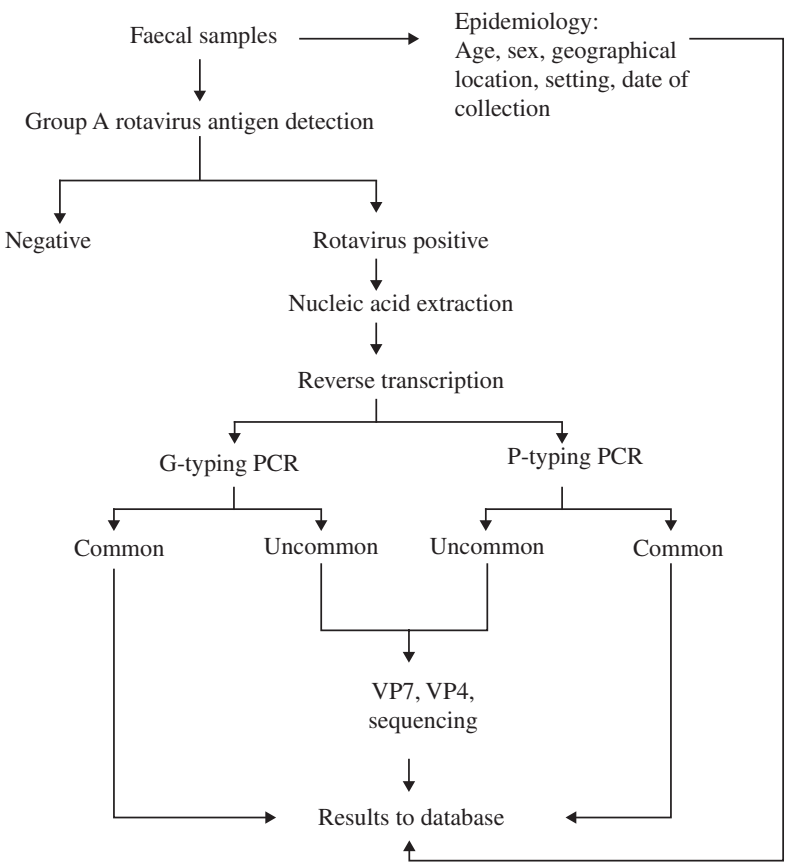

Fig. 1. Algorithm describing the testing and reporting of rotavirus-positive samples.
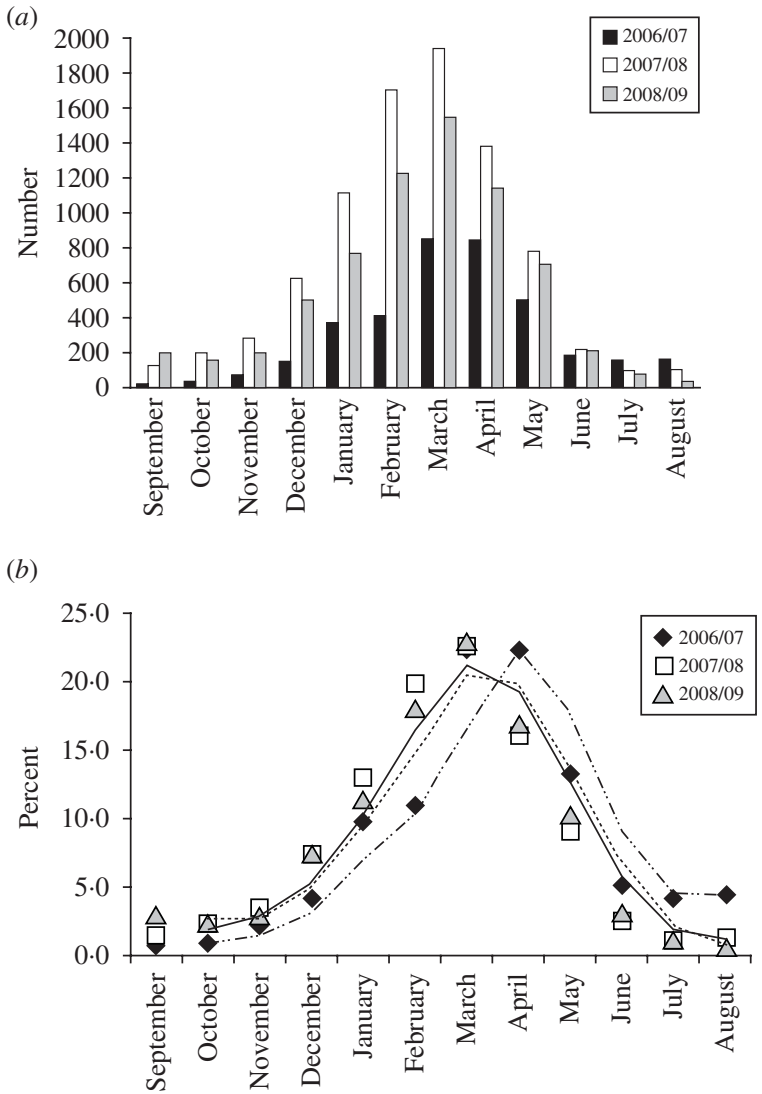

Fig. 2. Temporal distribution of rotavirus infections in the EuroRotaNet database in three consecutive seasons, 2006/2007, 2007/2008 and 2008/2009. (a) Numbers of strains each month in three consecutive seasons. (b) Percent of the total rotavirus strains in a season each month showing the moving-average trend analysis.

rotavirus detection and characterization methods $\mathrm{v} 4$ (http://www.eurorota.net/docs.php)] and following the algorithm described in Figure 1. Rotavirus subgroups (VP6) and NSP4 genotypes were determined for uncommon or novel strains identified through Gand P-typing in order to identify possible zoonotic transmission [18, 19] (European rotavirus detection and characterization methods v4).

\section{Epidemiological data}

Epidemiological data including age, sex, geographical location, including postcode in some countries, setting (hospital or community, urban or rural), symptoms (diarrhoea, vomiting, or diarrhoea and vomiting, or other) and date of onset and date of sample collection were entered into a web-accessible database and linked to the genotyping data (http:// www.eurorota.net/). 
Table 2. Possible origins of rotavirus strains circulating within Europe. Common and reassortant human rotavirus strains, zoonotic strains and animal-human hybrid rotavirus strains are identified

\begin{tabular}{|c|c|c|c|c|c|c|c|c|}
\hline & \multicolumn{2}{|c|}{$2006 / 2007$} & \multicolumn{2}{|c|}{$2007 / 2008$} & \multicolumn{2}{|c|}{$2008 / 2009$} & \multicolumn{2}{|l|}{ Total } \\
\hline & No. & $\%$ & No. & $\%$ & No. & $\%$ & No. & $\%$ \\
\hline \multicolumn{9}{|c|}{ Common human rotavirus genotypes } \\
\hline G1P $[8]$ & 1637 & $43 \cdot 00$ & 4532 & $52 \cdot 95$ & 3101 & $45 \cdot 78$ & 9270 & $48 \cdot 43$ \\
\hline $\mathrm{G} 2 \mathrm{P}[4]$ & 584 & $15 \cdot 34$ & 747 & $8 \cdot 73$ & 597 & $8 \cdot 81$ & 1928 & $10 \cdot 07$ \\
\hline G3P[8] & 113 & $2 \cdot 97$ & 324 & $3 \cdot 79$ & 381 & $5 \cdot 62$ & 818 & $4 \cdot 27$ \\
\hline G4P[8] & 282 & $7 \cdot 41$ & 1282 & $14 \cdot 98$ & 1318 & $19 \cdot 46$ & 2882 & $15 \cdot 06$ \\
\hline G9P[8] & 768 & $20 \cdot 17$ & 900 & $10 \cdot 52$ & 544 & $8 \cdot 03$ & 2212 & $11 \cdot 56$ \\
\hline \multicolumn{9}{|c|}{ Reassortment among common human rotavirus genotypes } \\
\hline G1P $[4]$ & 11 & $0 \cdot 29$ & 30 & $0 \cdot 35$ & 15 & $0 \cdot 22$ & 56 & $0 \cdot 29$ \\
\hline $\mathrm{G} 2 \mathrm{P}[8]$ & 21 & $0 \cdot 55$ & 46 & $0 \cdot 54$ & 23 & $0 \cdot 34$ & 90 & $0 \cdot 47$ \\
\hline G3P[4] & 1 & $0 \cdot 03$ & 1 & $0 \cdot 01$ & 5 & $0 \cdot 07$ & 7 & $0 \cdot 04$ \\
\hline $\mathrm{G} 4 \mathrm{P}[4]$ & 1 & $0 \cdot 03$ & 6 & $0 \cdot 07$ & 17 & $0 \cdot 25$ & 24 & $0 \cdot 13$ \\
\hline G9P[4] & 4 & $0 \cdot 11$ & 16 & $0 \cdot 19$ & 16 & $0 \cdot 24$ & 36 & $0 \cdot 19$ \\
\hline \multicolumn{9}{|c|}{ Potential zoonotic rotavirus genotypes } \\
\hline G3P[3] & 1 & 0.03 & 0 & $0 \cdot 00$ & 0 & $0 \cdot 00$ & 1 & $0 \cdot 01$ \\
\hline G3P[6] & 0 & $0 \cdot 00$ & 0 & $0 \cdot 00$ & 9 & $0 \cdot 13$ & 9 & $0 \cdot 05$ \\
\hline G3P[9] & 0 & $0 \cdot 00$ & 2 & $0 \cdot 02$ & 3 & $0 \cdot 04$ & 5 & 0.03 \\
\hline G6P[9] & 4 & $0 \cdot 11$ & 3 & $0 \cdot 04$ & 1 & $0 \cdot 01$ & 8 & 0.04 \\
\hline G6P[14] & 1 & $0 \cdot 03$ & 3 & $0 \cdot 04$ & 0 & $0 \cdot 00$ & 4 & 0.02 \\
\hline G8P[6] & 4 & $0 \cdot 11$ & 1 & $0 \cdot 01$ & 1 & $0 \cdot 01$ & 6 & 0.03 \\
\hline G8P[14] & 0 & $0 \cdot 00$ & 1 & $0 \cdot 01$ & 2 & $0 \cdot 01$ & 3 & 0.01 \\
\hline G9P[6] & 5 & $0 \cdot 13$ & 0 & $0 \cdot 00$ & 4 & $0 \cdot 06$ & 9 & 0.05 \\
\hline G9P[9] & 2 & $0 \cdot 05$ & 3 & $0 \cdot 04$ & 1 & $0 \cdot 01$ & 6 & 0.03 \\
\hline G9P[10] & 0 & $0 \cdot 00$ & 1 & $0 \cdot 01$ & 1 & $0 \cdot 01$ & 2 & 0.01 \\
\hline G10P[14] & 5 & $0 \cdot 13$ & 1 & $0 \cdot 01$ & 0 & $0 \cdot 00$ & 6 & 0.03 \\
\hline G12P[9] & 0 & $0 \cdot 00$ & 4 & $0 \cdot 05$ & 4 & $0 \cdot 06$ & 8 & 0.04 \\
\hline \multicolumn{9}{|c|}{ Possible human-animal hybrid rotavirus genotypes } \\
\hline G1P[6] & 0 & $0 \cdot 00$ & 0 & $0 \cdot 00$ & 4 & $0 \cdot 06$ & 4 & $0 \cdot 02$ \\
\hline $\mathrm{G} 2 \mathrm{P}[6]$ & 5 & $0 \cdot 13$ & 17 & $0 \cdot 20$ & 2 & $0 \cdot 03$ & 24 & $0 \cdot 13$ \\
\hline $\mathrm{G} 2 \mathrm{P}[9]$ & 1 & $0 \cdot 03$ & 1 & $0 \cdot 01$ & 0 & $0 \cdot 00$ & 2 & $0 \cdot 01$ \\
\hline $\mathrm{G} 2 \mathrm{P}[10]$ & 1 & $0 \cdot 03$ & 0 & $0 \cdot 00$ & 0 & $0 \cdot 00$ & 1 & $0 \cdot 01$ \\
\hline G4P[6] & 5 & $0 \cdot 13$ & 0 & $0 \cdot 00$ & 3 & $0 \cdot 04$ & 8 & 0.04 \\
\hline G4P[9] & 0 & $0 \cdot 00$ & 1 & $0 \cdot 01$ & 1 & $0 \cdot 01$ & 2 & $0 \cdot 01$ \\
\hline G4P[10] & 0 & $0 \cdot 00$ & 0 & $0 \cdot 00$ & 1 & $0 \cdot 01$ & 1 & 0.01 \\
\hline G6P[4] & 0 & $0 \cdot 00$ & 1 & $0 \cdot 01$ & 0 & $0 \cdot 00$ & 1 & $0 \cdot 01$ \\
\hline G6P[8] & 0 & $0 \cdot 00$ & 2 & $0 \cdot 02$ & 0 & 0.00 & 2 & 0.01 \\
\hline G6P[11] & 1 & $0 \cdot 03$ & 0 & $0 \cdot 00$ & 0 & $0 \cdot 00$ & 1 & 0.01 \\
\hline G8P[4] & 0 & $0 \cdot 00$ & 3 & $0 \cdot 04$ & 63 & 0.93 & 66 & $0 \cdot 34$ \\
\hline G8P[8] & 2 & $0 \cdot 05$ & 2 & $0 \cdot 02$ & 6 & 0.09 & 10 & 0.05 \\
\hline G10P[4] & 1 & $0 \cdot 03$ & 2 & $0 \cdot 02$ & 0 & $0 \cdot 00$ & 3 & 0.02 \\
\hline G10P[6] & 0 & $0 \cdot 00$ & 1 & $0 \cdot 01$ & 0 & $0 \cdot 00$ & 1 & $0 \cdot 01$ \\
\hline G10P[8] & 0 & $0 \cdot 00$ & 6 & 0.07 & 15 & $0 \cdot 22$ & 21 & $0 \cdot 11$ \\
\hline G10P[10] & 0 & $0 \cdot 00$ & 1 & $0 \cdot 01$ & 0 & $0 \cdot 00$ & 1 & 0.01 \\
\hline G12P[4] & 0 & $0 \cdot 00$ & 0 & $0 \cdot 00$ & 3 & $0 \cdot 04$ & 3 & $0 \cdot 02$ \\
\hline G12P[6] & 1 & $0 \cdot 03$ & 4 & $0 \cdot 05$ & 41 & $0 \cdot 61$ & 46 & $0 \cdot 24$ \\
\hline G12P[8] & 21 & $0 \cdot 55$ & 46 & $0 \cdot 54$ & 52 & $0 \cdot 77$ & 119 & $0 \cdot 62$ \\
\hline Total & 3482 & $91 \cdot 46$ & 7990 & $93 \cdot 35$ & 6234 & $92 \cdot 03$ & 17706 & $92 \cdot 51$ \\
\hline
\end{tabular}

\section{RESULTS}

\section{Temporal distribution}

Rotavirus infections peak in the winter months in temperate climates, and the analysis of the data in EuroRotaNet reflects this seasonality. The peak of rotavirus infections in Europe occurred in March in all three seasons between 2006 and 2009 (Fig. 2). The moving-average trend analysis indicated that rotavirus infections peaked in April in 
Table 3 (a). Co-infections with multiple rotavirus strains (single G-type with two or more P-types)

\begin{tabular}{|c|c|c|c|c|c|c|c|c|}
\hline & \multicolumn{2}{|c|}{$2006 / 2007$} & \multicolumn{2}{|c|}{$2007 / 2008$} & \multicolumn{2}{|c|}{$2008 / 2009$} & \multicolumn{2}{|c|}{ Total } \\
\hline & No. & $\%$ & No. & $\%$ & No. & $\%$ & No. & $\%$ \\
\hline $\mathrm{G} 1 \mathrm{P}[4]+\mathrm{P}[8]$ & 10 & $0 \cdot 26$ & 19 & $0 \cdot 22$ & 22 & $0 \cdot 32$ & 51 & $0 \cdot 27$ \\
\hline $\mathrm{G} 2 \mathrm{P}[4]+\mathrm{P}[8]$ & 6 & $0 \cdot 16$ & 6 & $0 \cdot 07$ & 12 & $0 \cdot 18$ & 24 & $0 \cdot 13$ \\
\hline G9P $[4]+P[8]$ & 6 & $0 \cdot 16$ & 10 & $0 \cdot 12$ & 8 & $0 \cdot 12$ & 24 & $0 \cdot 13$ \\
\hline $\mathrm{G} 9 \mathrm{P}[6]+\mathrm{P}[8]$ & 1 & $0 \cdot 03$ & 6 & $0 \cdot 07$ & 2 & $0 \cdot 03$ & 9 & $0 \cdot 05$ \\
\hline $\mathrm{G} 4 \mathrm{P}[4]+\mathrm{P}[8]$ & 1 & $0 \cdot 03$ & 2 & $0 \cdot 02$ & 3 & $0 \cdot 04$ & 6 & 0.03 \\
\hline $\mathrm{G} 3 \mathrm{P}[4]+\mathrm{P}[8]$ & 3 & $0 \cdot 08$ & 1 & $0 \cdot 01$ & 1 & $0 \cdot 01$ & 5 & 0.03 \\
\hline$G 8 P[4]+P[6]$ & 0 & $0 \cdot 00$ & 0 & $0 \cdot 00$ & 5 & 0.07 & 5 & $0 \cdot 03$ \\
\hline $\mathrm{G} 1 \mathrm{P}[6]+\mathrm{P}[8]$ & 1 & $0 \cdot 03$ & 1 & $0 \cdot 01$ & 1 & $0 \cdot 01$ & 3 & 0.02 \\
\hline $\mathrm{G} 10 \mathrm{P}[4]+\mathrm{P}[8]$ & 0 & $0 \cdot 00$ & 1 & $0 \cdot 01$ & 1 & $0 \cdot 01$ & 2 & $0 \cdot 01$ \\
\hline $\mathrm{G} 12 \mathrm{P}[4]+\mathrm{P}[8]$ & 0 & $0 \cdot 00$ & 1 & $0 \cdot 01$ & 1 & $0 \cdot 01$ & 2 & $0 \cdot 01$ \\
\hline $\mathrm{G} 1 \mathrm{P}[8]+\mathrm{P}[10]$ & 0 & $0 \cdot 00$ & 1 & $0 \cdot 01$ & 1 & $0 \cdot 01$ & 2 & $0 \cdot 01$ \\
\hline $\mathrm{G} 2 \mathrm{P}[4]+\mathrm{P}[6]$ & 1 & $0 \cdot 03$ & 0 & $0 \cdot 00$ & 1 & $0 \cdot 01$ & 2 & $0 \cdot 01$ \\
\hline $\mathrm{G} 2 \mathrm{P}[6]+\mathrm{P}[8]$ & 2 & $0 \cdot 05$ & 0 & $0 \cdot 00$ & 0 & $0 \cdot 00$ & 2 & $0 \cdot 01$ \\
\hline $\mathrm{G} 8 \mathrm{P}[6]+\mathrm{P}[8]$ & 0 & $0 \cdot 00$ & 0 & $0 \cdot 00$ & 2 & $0 \cdot 03$ & 2 & $0 \cdot 01$ \\
\hline $\mathrm{G} 12 \mathrm{P}[8]+\mathrm{P}[9]$ & 0 & $0 \cdot 00$ & 0 & $0 \cdot 00$ & 1 & $0 \cdot 01$ & 1 & $0 \cdot 01$ \\
\hline $\mathrm{G} 12 \mathrm{P}[9]+\mathrm{P}[11]$ & 0 & $0 \cdot 00$ & 0 & $0 \cdot 00$ & 1 & $0 \cdot 01$ & 1 & $0 \cdot 01$ \\
\hline $\mathrm{G} 1 \mathrm{P}[4]+\mathrm{P}[6]+\mathrm{P}[8]$ & 0 & $0 \cdot 00$ & 1 & $0 \cdot 01$ & 0 & $0 \cdot 00$ & 1 & $0 \cdot 01$ \\
\hline $\mathrm{G} 1 \mathrm{P}[8]+\mathrm{P}[9]$ & 0 & $0 \cdot 00$ & 1 & $0 \cdot 01$ & 0 & $0 \cdot 00$ & 1 & $0 \cdot 01$ \\
\hline $\mathrm{G} 2 \mathrm{P}[4]+\mathrm{P}[9]$ & 1 & $0 \cdot 03$ & 0 & $0 \cdot 00$ & 0 & $0 \cdot 00$ & 1 & $0 \cdot 01$ \\
\hline $\mathrm{G} 3 \mathrm{P}[4]+\mathrm{P}[6]$ & 0 & $0 \cdot 00$ & 0 & $0 \cdot 00$ & 1 & $0 \cdot 01$ & 1 & $0 \cdot 01$ \\
\hline $\mathrm{G} 4 \mathrm{P}[4]+\mathrm{P}[6]$ & 0 & $0 \cdot 00$ & 0 & $0 \cdot 00$ & 1 & $0 \cdot 01$ & 1 & $0 \cdot 01$ \\
\hline $\mathrm{G} 8 \mathrm{P}[4]+\mathrm{P}[8]$ & 0 & $0 \cdot 00$ & 0 & $0 \cdot 00$ & 1 & $0 \cdot 01$ & 1 & 0.01 \\
\hline $\mathrm{G} 9 \mathrm{P}[8]+\mathrm{P}[9]$ & 1 & $0 \cdot 03$ & 0 & $0 \cdot 00$ & 0 & $0 \cdot 00$ & 1 & $0 \cdot 01$ \\
\hline Total & 33 & $0 \cdot 87$ & 50 & $0 \cdot 58$ & 65 & $0 \cdot 96$ & 148 & $0 \cdot 77$ \\
\hline
\end{tabular}

2006/2007 and in March in 2007/2008 and 2008/2009

(Fig. 2).

Differences were observed across EuroRotaNet participating countries in the month in which rotavirus infections peaked. The earliest peaks of infection were detected in Spain in 2006/2007 and 2008/2009, in January and December, respectively, and in 2007/ 2008 infection peaked in February. Late season peaks were associated with countries in the East or North of Europe with infection peaking in Hungary in May 2007 and in Finland in May 2008 and 2009.

\section{Genotype distribution}

\section{Total dataset}

The total number of rotavirus-positive samples included were 19140 for the 3 years and included 3807 in 2006/2007, 8559 in 2007/2008, and 6774 in 2008/ 2009. These numbers were used as denominators for calculating percentages. A total of 141 different combinations of G- and P-types were found between 2006 and 2009 and included those with combinations either singly or as multiple infections of G1, G2, G3, G4,
G6, G8, G9, G10, G12 and P[3], P[4], P[6], P[8], P[9], $\mathrm{P}[10], \mathrm{P}[11], \mathrm{P}[14]$ genotypes (Table 2).

\section{Common rotavirus strains}

$\mathrm{G} 1 \mathrm{P}[8]$ rotavirus strains were predominant in all three seasons between 2006 and 2009. Strains circulating with a prevalence of $>1 \%$ included G1P $[8](48.43 \%)$, G4P[8] (15.06\%), G9P[8] (11.56\%), G2P[4] (10.07\%) and G3P[8] (4.27\%) (Table 2).

\section{Possible origins of rotavirus strains}

Rotavirus strains found circulating within the European population between 2006 and 2009 can be stratified according to their possible origins. Human rotavirus strains are the most prevalent, making up $89.4 \%$ of circulating strains. Strains whose derivation may be associated with reassortment between animal and human strains make up $1.7 \%$ and reassortants of the common human strains make up $1 \cdot 1 \%$. Infection with rotavirus strains of animal origin may represent $0 \cdot 3 \%$ of strains found (Table 2 ). 
Table $3(b)$. Co-infections with multiple rotavirus strains (multiple G-types with a single P-type)

\begin{tabular}{|c|c|c|c|c|c|c|c|c|}
\hline & \multicolumn{2}{|c|}{$2006 / 2007$} & \multicolumn{2}{|c|}{$2007 / 2008$} & \multicolumn{2}{|c|}{$2008 / 2009$} & \multicolumn{2}{|c|}{ Total } \\
\hline & No. & $\%$ & No. & $\%$ & No. & $\%$ & No. & $\%$ \\
\hline G1 + G9P[8] & 70 & $1 \cdot 84$ & 103 & $1 \cdot 20$ & 34 & $0 \cdot 50$ & 207 & $1 \cdot 08$ \\
\hline $\mathrm{G} 1+\mathrm{G} 4 \mathrm{P}[8]$ & 5 & $0 \cdot 13$ & 36 & $0 \cdot 42$ & 36 & $0 \cdot 53$ & 77 & $0 \cdot 40$ \\
\hline $\mathrm{G} 1+\mathrm{G} 2 \mathrm{P}[4]$ & 5 & $0 \cdot 13$ & 22 & $0 \cdot 26$ & 9 & $0 \cdot 13$ & 36 & $0 \cdot 19$ \\
\hline G4 + G9P[8] & 1 & $0 \cdot 03$ & 12 & $0 \cdot 14$ & 19 & $0 \cdot 28$ & 32 & $0 \cdot 17$ \\
\hline G3 + G9P[8] & 8 & $0 \cdot 21$ & 12 & $0 \cdot 14$ & 10 & $0 \cdot 15$ & 30 & $0 \cdot 16$ \\
\hline $\mathrm{G} 1+\mathrm{G} 3 \mathrm{P}[8]$ & 5 & $0 \cdot 13$ & 10 & $0 \cdot 12$ & 7 & $0 \cdot 10$ & 22 & $0 \cdot 11$ \\
\hline $\mathrm{G} 1+\mathrm{G} 2 \mathrm{P}[8]$ & 9 & $0 \cdot 24$ & 4 & $0 \cdot 05$ & 2 & 0.03 & 15 & 0.08 \\
\hline $\mathrm{G} 1+\mathrm{G} 10 \mathrm{P}[8]$ & 0 & $0 \cdot 00$ & 9 & $0 \cdot 11$ & 2 & 0.03 & 11 & 0.06 \\
\hline $\mathrm{G} 1+\mathrm{G} 12 \mathrm{P}[8]$ & 2 & $0 \cdot 05$ & 2 & $0 \cdot 02$ & 5 & $0 \cdot 07$ & 9 & 0.05 \\
\hline $\mathrm{G} 2+\mathrm{G} 4 \mathrm{P}[8]$ & 4 & $0 \cdot 11$ & 0 & $0 \cdot 00$ & 5 & $0 \cdot 07$ & 9 & 0.05 \\
\hline $\mathrm{G} 1+\mathrm{G} 2+\mathrm{G} 9 \mathrm{P}[8]$ & 8 & $0 \cdot 21$ & 0 & $0 \cdot 00$ & 0 & $0 \cdot 00$ & 8 & $0 \cdot 04$ \\
\hline $\mathrm{G} 2+\mathrm{G} 4 \mathrm{P}[4]$ & 0 & $0 \cdot 00$ & 3 & $0 \cdot 04$ & 5 & $0 \cdot 07$ & 8 & $0 \cdot 04$ \\
\hline $\mathrm{G} 2+\mathrm{G} 9 \mathrm{P}[4]$ & 4 & $0 \cdot 11$ & 2 & $0 \cdot 02$ & 2 & $0 \cdot 03$ & 8 & $0 \cdot 04$ \\
\hline $\mathrm{G} 3+\mathrm{G} 8 \mathrm{P}[8]$ & 0 & $0 \cdot 00$ & 0 & $0 \cdot 00$ & 6 & 0.09 & 6 & $0 \cdot 03$ \\
\hline $\mathrm{G} 9+\mathrm{G} 12 \mathrm{P}[8]$ & 0 & $0 \cdot 00$ & 1 & $0 \cdot 01$ & 4 & $0 \cdot 06$ & 5 & 0.03 \\
\hline $\mathrm{G} 2+\mathrm{G} 9 \mathrm{P}[8]$ & 3 & $0 \cdot 08$ & 0 & $0 \cdot 00$ & 1 & $0 \cdot 01$ & 4 & 0.02 \\
\hline $\mathrm{G} 3+\mathrm{G} 4 \mathrm{P}[8]$ & 1 & $0 \cdot 03$ & 0 & $0 \cdot 00$ & 3 & $0 \cdot 04$ & 4 & $0 \cdot 02$ \\
\hline $\mathrm{G} 1+\mathrm{G} 3+\mathrm{G} 9 \mathrm{P}[8]$ & 3 & $0 \cdot 08$ & 0 & $0 \cdot 00$ & 0 & $0 \cdot 00$ & 3 & $0 \cdot 02$ \\
\hline $\mathrm{G} 1+\mathrm{G} 9 \mathrm{P}[4]$ & 2 & $0 \cdot 05$ & 1 & $0 \cdot 01$ & 0 & $0 \cdot 00$ & 3 & $0 \cdot 02$ \\
\hline $\mathrm{G} 4+\mathrm{G} 12 \mathrm{P}[8]$ & 0 & $0 \cdot 00$ & 2 & $0 \cdot 02$ & 1 & $0 \cdot 01$ & 3 & 0.02 \\
\hline $\mathrm{G} 1+\mathrm{G} 4+\mathrm{G} 9 \mathrm{P}[8]$ & 1 & $0 \cdot 03$ & 1 & $0 \cdot 01$ & 0 & $0 \cdot 00$ & 2 & $0 \cdot 01$ \\
\hline G1 + G9P[6] & 1 & $0 \cdot 03$ & 0 & $0 \cdot 00$ & 1 & $0 \cdot 01$ & 2 & $0 \cdot 01$ \\
\hline $\mathrm{G} 2+\mathrm{G} 12 \mathrm{P}[4]$ & 0 & $0 \cdot 00$ & 0 & $0 \cdot 00$ & 2 & $0 \cdot 03$ & 2 & $0 \cdot 01$ \\
\hline $\mathrm{G} 2+\mathrm{G} 3 \mathrm{P}[4]$ & 0 & $0 \cdot 00$ & 0 & $0 \cdot 00$ & 2 & 0.03 & 2 & $0 \cdot 01$ \\
\hline $\mathrm{G} 3+\mathrm{G} 9+\mathrm{G} 12 \mathrm{P}[8]$ & 0 & $0 \cdot 00$ & 2 & $0 \cdot 02$ & 0 & $0 \cdot 00$ & 2 & $0 \cdot 01$ \\
\hline $\mathrm{G} 4+\mathrm{G} 8 \mathrm{P}[4]$ & 0 & $0 \cdot 00$ & 0 & $0 \cdot 00$ & 2 & $0 \cdot 03$ & 2 & $0 \cdot 01$ \\
\hline $\mathrm{G} 9+\mathrm{G} 10 \mathrm{P}[8]$ & 0 & $0 \cdot 00$ & 0 & $0 \cdot 00$ & 2 & $0 \cdot 03$ & 2 & $0 \cdot 01$ \\
\hline $\mathrm{G} 1+\mathrm{G} 12 \mathrm{P}[4]$ & 0 & $0 \cdot 00$ & 0 & $0 \cdot 00$ & 1 & $0 \cdot 01$ & 1 & $0 \cdot 01$ \\
\hline $\mathrm{G} 1+\mathrm{G} 12 \mathrm{P}[6]$ & 1 & $0 \cdot 03$ & 0 & $0 \cdot 00$ & 0 & $0 \cdot 00$ & 1 & $0 \cdot 01$ \\
\hline $\mathrm{G} 1+\mathrm{G} 2+\mathrm{G} 3 \mathrm{P}[8]$ & 1 & $0 \cdot 03$ & 0 & $0 \cdot 00$ & 0 & $0 \cdot 00$ & 1 & $0 \cdot 01$ \\
\hline $\mathrm{G} 1+\mathrm{G} 2+\mathrm{G} 4 \mathrm{P}[8]$ & 0 & $0 \cdot 00$ & 0 & $0 \cdot 00$ & 1 & $0 \cdot 01$ & 1 & $0 \cdot 01$ \\
\hline $\mathrm{G} 1+\mathrm{G} 2+\mathrm{G} 9 \mathrm{P}[4]$ & 1 & $0 \cdot 03$ & 0 & $0 \cdot 00$ & 0 & $0 \cdot 00$ & 1 & $0 \cdot 01$ \\
\hline G1 + G6P[9] & 0 & $0 \cdot 00$ & 1 & $0 \cdot 01$ & 0 & $0 \cdot 00$ & 1 & $0 \cdot 01$ \\
\hline $\mathrm{G} 1+\mathrm{G} 8+\mathrm{G} 12 \mathrm{P}[8]$ & 0 & $0 \cdot 00$ & 0 & $0 \cdot 00$ & 1 & $0 \cdot 01$ & 1 & $0 \cdot 01$ \\
\hline $\mathrm{G} 1+\mathrm{G} 8 \mathrm{P}[4]$ & 0 & $0 \cdot 00$ & 0 & $0 \cdot 00$ & 1 & $0 \cdot 01$ & 1 & $0 \cdot 01$ \\
\hline $\mathrm{G} 1$ + G8P[8] & 0 & $0 \cdot 00$ & 0 & $0 \cdot 00$ & 1 & $0 \cdot 01$ & 1 & $0 \cdot 01$ \\
\hline $\mathrm{G} 10+\mathrm{G} 12 \mathrm{P}[6]$ & 0 & $0 \cdot 00$ & 0 & $0 \cdot 00$ & 1 & $0 \cdot 01$ & 1 & $0 \cdot 01$ \\
\hline $\mathrm{G} 2+\mathrm{G} 10 \mathrm{P}[4]$ & 0 & $0 \cdot 00$ & 1 & $0 \cdot 01$ & 0 & $0 \cdot 00$ & 1 & $0 \cdot 01$ \\
\hline $\mathrm{G} 2+\mathrm{G} 9 \mathrm{P}[6]$ & 0 & $0 \cdot 00$ & 1 & $0 \cdot 01$ & 0 & $0 \cdot 00$ & 1 & $0 \cdot 01$ \\
\hline $\mathrm{G} 3+\mathrm{G} 10 \mathrm{P}[8]$ & 0 & $0 \cdot 00$ & 1 & $0 \cdot 01$ & 0 & $0 \cdot 00$ & 1 & $0 \cdot 01$ \\
\hline $\mathrm{G} 3+\mathrm{G} 8 \mathrm{P}[8]$ & 0 & $0 \cdot 00$ & 1 & $0 \cdot 01$ & 0 & $0 \cdot 00$ & 1 & $0 \cdot 01$ \\
\hline $\mathrm{G} 3+\mathrm{G} 9 \mathrm{P}[4]$ & 0 & $0 \cdot 00$ & 1 & $0 \cdot 01$ & 0 & $0 \cdot 00$ & 1 & $0 \cdot 01$ \\
\hline $\mathrm{G} 3+\mathrm{G} 9 \mathrm{P}[6]$ & 0 & $0 \cdot 00$ & 1 & $0 \cdot 01$ & 0 & $0 \cdot 00$ & 1 & $0 \cdot 01$ \\
\hline G3 + G9P[9] & 1 & $0 \cdot 03$ & 0 & $0 \cdot 00$ & 0 & $0 \cdot 00$ & 1 & $0 \cdot 01$ \\
\hline $\mathrm{G} 4+\mathrm{G} 10 \mathrm{P}[8]$ & 0 & $0 \cdot 00$ & 0 & $0 \cdot 00$ & 1 & $0 \cdot 01$ & 1 & $0 \cdot 01$ \\
\hline $\mathrm{G} 8+\mathrm{G} 12 \mathrm{P}[4]$ & 0 & $0 \cdot 00$ & 0 & $0 \cdot 00$ & 1 & $0 \cdot 01$ & 1 & $0 \cdot 01$ \\
\hline $\mathrm{G} 9+\mathrm{G} 3 \mathrm{P}[6]$ & 0 & $0 \cdot 00$ & 1 & $0 \cdot 01$ & 0 & $0 \cdot 00$ & 1 & $0 \cdot 01$ \\
\hline Total & 136 & $3 \cdot 57$ & 230 & $2 \cdot 69$ & 168 & $2 \cdot 48$ & 534 & $2 \cdot 79$ \\
\hline
\end{tabular}

\section{Mixed rotavirus infections}

A total of 809 mixed rotavirus infections were detected between 2006 and 2009. These could be classified as single G-type with two or more P-types (148, $0 \cdot 8 \%$ ), multiple G-types with a single P-type (534, $2 \cdot 8 \%$ ) or multiple G- and P-types (127, $0 \cdot 7 \%$ ). These strains are representative of those genotypes 
Table $3(c)$. Co-infections with multiple rotavirus strains (multiple $G$ - and P-types)

\begin{tabular}{|c|c|c|c|c|c|c|c|c|}
\hline & \multicolumn{2}{|c|}{$2006 / 2007$} & \multicolumn{2}{|c|}{$2007 / 2008$} & \multicolumn{2}{|c|}{$2008 / 2009$} & \multicolumn{2}{|c|}{ Total } \\
\hline & No. & $\%$ & No. & $\%$ & No. & $\%$ & No. & $\%$ \\
\hline $\mathrm{G} 1+\mathrm{G} 2 \mathrm{P}[4]+\mathrm{P}[8]$ & 14 & $0 \cdot 37$ & 18 & $0 \cdot 21$ & 10 & $0 \cdot 15$ & 42 & $0 \cdot 22$ \\
\hline $\mathrm{G} 2+\mathrm{G} 9 \mathrm{P}[4]+\mathrm{P}[8]$ & 7 & $0 \cdot 18$ & 9 & $0 \cdot 11$ & 6 & $0 \cdot 09$ & 22 & $0 \cdot 11$ \\
\hline $\mathrm{G} 2+\mathrm{G} 4 \mathrm{P}[4]+\mathrm{P}[8]$ & 0 & $0 \cdot 00$ & 2 & $0 \cdot 02$ & 6 & $0 \cdot 09$ & 8 & $0 \cdot 04$ \\
\hline $\mathrm{G} 1+\mathrm{G} 2+\mathrm{G} 9 \mathrm{P}[4]+\mathrm{P}[8]$ & 5 & $0 \cdot 13$ & 1 & $0 \cdot 01$ & 1 & $0 \cdot 01$ & 7 & $0 \cdot 04$ \\
\hline $\mathrm{G} 1+\mathrm{G} 9 \mathrm{P}[4]+\mathrm{P}[8]$ & 3 & $0 \cdot 08$ & 3 & $0 \cdot 04$ & 1 & $0 \cdot 01$ & 7 & $0 \cdot 04$ \\
\hline $\mathrm{G} 2+\mathrm{G} 3 \mathrm{P}[4]+\mathrm{P}[8]$ & 2 & $0 \cdot 05$ & 0 & $0 \cdot 00$ & 4 & $0 \cdot 06$ & 6 & $0 \cdot 03$ \\
\hline $\mathrm{G} 1+\mathrm{G} 4 \mathrm{P}[4]+\mathrm{P}[8]$ & 0 & $0 \cdot 00$ & 0 & $0 \cdot 00$ & 5 & $0 \cdot 07$ & 5 & $0 \cdot 03$ \\
\hline $\mathrm{G} 1+\mathrm{G} 9 \mathrm{P}[6]+\mathrm{P}[8]$ & 3 & $0 \cdot 08$ & 0 & $0 \cdot 00$ & 0 & $0 \cdot 00$ & 3 & $0 \cdot 02$ \\
\hline $\mathrm{G} 4+\mathrm{G} 9 \mathrm{P}[4]+\mathrm{P}[8]$ & 0 & $0 \cdot 00$ & 0 & $0 \cdot 00$ & 3 & $0 \cdot 04$ & 3 & $0 \cdot 02$ \\
\hline $\mathrm{G} 1+\mathrm{G} 2 \mathrm{P}[4]+\mathrm{P}[6]$ & 0 & $0 \cdot 00$ & 1 & $0 \cdot 01$ & 1 & $0 \cdot 01$ & 2 & $0 \cdot 01$ \\
\hline $\mathrm{G} 1+\mathrm{G} 3 \mathrm{P}[4]+\mathrm{P}[8]$ & 0 & $0 \cdot 00$ & 1 & $0 \cdot 01$ & 1 & $0 \cdot 01$ & 2 & $0 \cdot 01$ \\
\hline $\mathrm{G} 2+\mathrm{G} 12 \mathrm{P}[4]+\mathrm{P}[8]$ & 1 & $0 \cdot 03$ & 1 & $0 \cdot 01$ & 0 & $0 \cdot 00$ & 2 & $0 \cdot 01$ \\
\hline $\mathrm{G} 8+\mathrm{G} 12 \mathrm{P}[6]+\mathrm{P}[8]$ & 0 & $0 \cdot 00$ & 0 & $0 \cdot 00$ & 2 & $0 \cdot 03$ & 2 & $0 \cdot 01$ \\
\hline $\mathrm{G} 1+\mathrm{G} 2+\mathrm{G} 12 \mathrm{P}[4]+\mathrm{P}[8]$ & 0 & $0 \cdot 00$ & 0 & $0 \cdot 00$ & 1 & $0 \cdot 01$ & 1 & $0 \cdot 01$ \\
\hline $\mathrm{G} 1+\mathrm{G} 2+\mathrm{G} 3 \mathrm{P}[4]+\mathrm{P}[8]$ & 1 & $0 \cdot 03$ & 0 & $0 \cdot 00$ & 0 & $0 \cdot 00$ & 1 & $0 \cdot 01$ \\
\hline $\mathrm{G} 1+\mathrm{G} 2+\mathrm{G} 4 \mathrm{P}[4]+\mathrm{P}[8]$ & 0 & $0 \cdot 00$ & 0 & $0 \cdot 00$ & 1 & $0 \cdot 01$ & 1 & $0 \cdot 01$ \\
\hline $\mathrm{G} 1+\mathrm{G} 2+\mathrm{G} 4 \mathrm{P}[8]+\mathrm{P}[10]$ & 0 & $0 \cdot 00$ & 0 & $0 \cdot 00$ & 1 & $0 \cdot 01$ & 1 & $0 \cdot 01$ \\
\hline $\mathrm{G} 1+\mathrm{G} 3 \mathrm{P}[6]+\mathrm{P}[8]$ & 0 & $0 \cdot 00$ & 1 & $0 \cdot 01$ & 0 & $0 \cdot 00$ & 1 & $0 \cdot 01$ \\
\hline $\mathrm{G} 1+\mathrm{G} 9 \mathrm{P}[8]+\mathrm{P}[9]$ & 0 & $0 \cdot 00$ & 0 & $0 \cdot 00$ & 1 & $0 \cdot 01$ & 1 & $0 \cdot 01$ \\
\hline $\mathrm{G} 2+\mathrm{G} 12 \mathrm{P}[6]+\mathrm{P}[8]$ & 0 & $0 \cdot 00$ & 0 & $0 \cdot 00$ & 1 & $0 \cdot 01$ & 1 & $0 \cdot 01$ \\
\hline $\mathrm{G} 2+\mathrm{G} 4 \mathrm{P}[6]+\mathrm{P}[8]$ & 0 & $0 \cdot 00$ & 0 & $0 \cdot 00$ & 1 & $0 \cdot 01$ & 1 & $0 \cdot 01$ \\
\hline $\mathrm{G} 2+\mathrm{G} 9 \mathrm{P}[4]+\mathrm{P}[6]$ & 0 & $0 \cdot 00$ & 0 & $0 \cdot 00$ & 1 & $0 \cdot 01$ & 1 & $0 \cdot 01$ \\
\hline $\mathrm{G} 3+\mathrm{G} 4+\mathrm{G} 9 \mathrm{P}[8]+\mathrm{P}[9]$ & 0 & $0 \cdot 00$ & 1 & $0 \cdot 01$ & 0 & $0 \cdot 00$ & 1 & $0 \cdot 01$ \\
\hline $\mathrm{G} 3+\mathrm{G} 4 \mathrm{P}[4]+\mathrm{P}[8]$ & 0 & $0 \cdot 00$ & 1 & $0 \cdot 01$ & 0 & $0 \cdot 00$ & 1 & $0 \cdot 01$ \\
\hline $\mathrm{G} 3+\mathrm{G} 9 \mathrm{P}[4]+\mathrm{P}[8]$ & 1 & $0 \cdot 03$ & 0 & $0 \cdot 00$ & 0 & $0 \cdot 00$ & 1 & $0 \cdot 01$ \\
\hline $\mathrm{G} 4+\mathrm{G} 12 \mathrm{P}[4]+\mathrm{P}[8]$ & 0 & $0 \cdot 00$ & 0 & $0 \cdot 00$ & 1 & $0 \cdot 01$ & 1 & $0 \cdot 01$ \\
\hline $\mathrm{G} 8+\mathrm{G} 12 \mathrm{P}[4]+\mathrm{P}[6]$ & 0 & $0 \cdot 00$ & 0 & $0 \cdot 00$ & 1 & $0 \cdot 01$ & 1 & $0 \cdot 01$ \\
\hline $\mathrm{G} 9+\mathrm{G} 12 \mathrm{P}[4]+\mathrm{P}[8]$ & 0 & $0 \cdot 00$ & 1 & $0 \cdot 01$ & 0 & $0 \cdot 00$ & 1 & $0 \cdot 01$ \\
\hline $\mathrm{G} 9+\mathrm{G} 12 \mathrm{P}[8]+\mathrm{P}[9]$ & 1 & $0 \cdot 03$ & 0 & $0 \cdot 00$ & 0 & $0 \cdot 00$ & 1 & $0 \cdot 01$ \\
\hline Total & 38 & $1 \cdot 00$ & 40 & $0 \cdot 47$ & 49 & $0 \cdot 72$ & 127 & 0.66 \\
\hline
\end{tabular}

seen in single infections and include mixtures of human and of animal rotavirus strains (Table $3 a-c$ ).

\section{Partially typed rotavirus strains}

A total of 625 strains were partially typed, in 330 $(1.72 \%)$ and $295(1.54 \%)$ strains, only the P-type or G-type, respectively, was obtained (Table 4).

\section{Emerging rotavirus strains}

Rotavirus strains emerging in Europe between 2006 and 2009 included G12 and G8 strains (Table 5). These genotypes were found circulating in each of the three seasons and both G12 $\left(\chi^{2}=22 \cdot 83, P<0.0001\right)$ and G8 $\left(\chi^{2}=69 \cdot 66, P<0.0001\right)$ showed significant increases in the third season.

\section{Changing molecular epidemiology}

G1P[8] strains were most prevalent in the $2007 / 2008$ season with the exception of Lithuania where only $5.5 \%$ of strains were of this genotype. Over the three seasons, and in the majority of countries, the number of G1P[8] strains rose in the second season and fell in the third. The highest incidences of infection with G2P[4] strains in any season were in Belgium, Bulgaria and Greece where this genotype was found in $>30 \%$ of the strains. In season $1, \mathrm{G} 2 \mathrm{P}[4]$ was the most prevalent strain in Bulgaria and in seasons 1 and 2 in Belgium. In the majority of countries, this genotype accounted for $<20 \%$ of strains in any season. G3P[8] accounted for $<20 \%$ of strains in all countries in any season. This strain was not found in the first season in Bulgaria, Italy or Hungary, in the second in Slovenia and in the third in Bulgaria 
Table 4. Partially typed rotavirus strains

\begin{tabular}{|c|c|c|c|c|c|c|c|c|}
\hline & \multicolumn{2}{|c|}{$2006 / 2007$} & \multicolumn{2}{|c|}{$2007 / 2008$} & \multicolumn{2}{|c|}{$2008 / 2009$} & \multicolumn{2}{|c|}{ Total } \\
\hline & No. & $\%$ & No. & $\%$ & No. & $\%$ & No. & $\%$ \\
\hline G-UDP[8] & 40 & $1 \cdot 05$ & 98 & $1 \cdot 14$ & 129 & $1 \cdot 90$ & 267 & $1 \cdot 39$ \\
\hline G-UDP[4] & 6 & $0 \cdot 16$ & 8 & 0.09 & 16 & $0 \cdot 24$ & 30 & $0 \cdot 16$ \\
\hline G-UDP [4] + P[8] & 3 & $0 \cdot 08$ & 4 & $0 \cdot 05$ & 4 & $0 \cdot 06$ & 11 & $0 \cdot 06$ \\
\hline G-UDP $[6]$ & 1 & $0 \cdot 03$ & 0 & 0.00 & 9 & $0 \cdot 13$ & 10 & 0.05 \\
\hline G-UDP[9] & 0 & $0 \cdot 00$ & 0 & 0.00 & 6 & $0 \cdot 09$ & 6 & $0 \cdot 03$ \\
\hline G-UDP[10] & 1 & $0 \cdot 03$ & 1 & $0 \cdot 01$ & 2 & $0 \cdot 03$ & 4 & $0 \cdot 02$ \\
\hline G-UDP[11] & 0 & $0 \cdot 00$ & 0 & $0 \cdot 00$ & 1 & $0 \cdot 01$ & 1 & $0 \cdot 01$ \\
\hline G-UDP[8] + P[14] & 0 & $0 \cdot 00$ & 0 & $0 \cdot 00$ & 1 & $0 \cdot 01$ & 1 & $0 \cdot 01$ \\
\hline Total & 51 & $1 \cdot 34$ & 111 & $1 \cdot 30$ & 168 & $2 \cdot 48$ & 330 & $1 \cdot 72$ \\
\hline G1P-UD & 24 & $0 \cdot 63$ & 85 & 0.99 & 40 & $0 \cdot 59$ & 149 & $0 \cdot 78$ \\
\hline G9P-UD & 17 & $0 \cdot 45$ & 11 & $0 \cdot 13$ & 13 & $0 \cdot 19$ & 41 & $0 \cdot 21$ \\
\hline G2P-UD & 15 & $0 \cdot 39$ & 13 & $0 \cdot 15$ & 7 & $0 \cdot 10$ & 35 & $0 \cdot 18$ \\
\hline G4P-UD & 4 & $0 \cdot 11$ & 11 & $0 \cdot 13$ & 7 & $0 \cdot 10$ & 22 & $0 \cdot 11$ \\
\hline G3P-UD & 3 & $0 \cdot 08$ & 7 & $0 \cdot 08$ & 10 & $0 \cdot 15$ & 20 & $0 \cdot 10$ \\
\hline G12P-UD & 2 & $0 \cdot 05$ & 2 & 0.02 & 6 & $0 \cdot 09$ & 10 & 0.05 \\
\hline G10P-UD & 0 & $0 \cdot 00$ & 3 & $0 \cdot 04$ & 2 & $0 \cdot 03$ & 5 & 0.03 \\
\hline G1 + G9P-UD & 0 & $0 \cdot 00$ & 3 & $0 \cdot 04$ & 1 & $0 \cdot 01$ & 4 & 0.02 \\
\hline G1 + G4P-UD & 1 & $0 \cdot 03$ & 1 & $0 \cdot 01$ & 2 & $0 \cdot 03$ & 4 & 0.02 \\
\hline G8P-UD & 0 & $0 \cdot 00$ & 1 & $0 \cdot 01$ & 1 & $0 \cdot 01$ & 2 & $0 \cdot 01$ \\
\hline $\mathrm{G} 1+\mathrm{G} 2+\mathrm{G} 9 \mathrm{P}-\mathrm{UD}$ & 1 & $0 \cdot 03$ & 0 & $0 \cdot 00$ & 0 & $0 \cdot 00$ & 1 & $0 \cdot 01$ \\
\hline $\mathrm{G} 1+\mathrm{G} 2 \mathrm{P}-\mathrm{UD}$ & 0 & $0 \cdot 00$ & 1 & $0 \cdot 01$ & 0 & $0 \cdot 00$ & 1 & $0 \cdot 01$ \\
\hline $\mathrm{G} 1+\mathrm{G} 3 \mathrm{P}-\mathrm{UD}$ & 0 & $0 \cdot 00$ & 0 & $0 \cdot 00$ & 1 & $0 \cdot 01$ & 1 & $0 \cdot 01$ \\
\hline Total & 67 & $1 \cdot 76$ & 138 & $1 \cdot 61$ & 90 & $1 \cdot 33$ & 295 & $1 \cdot 54$ \\
\hline
\end{tabular}

and Greece. The prevalence of G4P[8] strains was $80 \%$ in Lithuania in $2007 / 2008$ and $>40 \%$ in Bulgaria and Germany in 2008/2009, and was found in all countries in the third season (2008/2009). G9P[8] strains were the most common strain found in Spain in the first season $(2006 / 2007)$, and the second most common in Bulgaria, France, Italy and Finland (Fig. 3).

G12 rotavirus strains were found to be circulating with an incidence of $>1 \%$ in nine countries including Finland (6.8\%), Denmark (3.2\%), Lithuania (2.4\%), Hungary $(2.3 \%)$, Greece $(1.6 \%)$, The Netherlands $(1.8 \%)$, Germany $(1.7 \%)$, UK $(1.5 \%)$ and Bulgaria $(1.2 \%)$. With the exception of Sweden, G12 strains were found in all other countries. G12 strains were found in each of the three seasons in Denmark, Hungary, UK, Bulgaria, Italy and France.

G8 strains were found in two countries in the $2006 / 2007$ season, in seven countries in the 2007/ 2008 season and in nine countries in the 2008/2009 season, with the highest incidence overall seen in UK $(2 \cdot 1 \%)$. The incidence was $<1 \%$ in all other countries (Belgium, Bulgaria, Denmark, France, Germany, Greece, Italy, Lithuania, Spain, Sweden, The Netherlands).

\section{Demographic data}

\section{Age distribution}

Infection peaked in children aged 1-2 years, but was seen in all age groups (Fig. 4a). Minor peaks, possibly associated with contact with infected children or grandchildren and waning immunity in the elderly population could also be seen (Fig. $4 b$ ).

\section{Distribution according to hospital or community setting}

For those cases for whom the setting was provided, $62.4 \%$ of samples were collected from hospitalized patients and $37.6 \%$ from patients in the community. With the exception of G2P[4] strains there were no significant differences in the distribution of common or emerging genotypes between hospital patients and those in the community (Table $6 a$ ). G2P[4] was significantly associated $\left(\chi^{2}=22.98, P<0.0001\right)$ with hospitalized patients and may be associated with the periodic upsurge of G2P[4] strains, possibly associated with the selection of an antibody escape mutant. Infection can often require hospitalization as symptoms may be severe in the absence of cross-reactive antibodies associated with previous infections (see Age distribution section above). 
Table 5. Distribution of possible emerging strains between 2006 and 2009 as single strain infections or contributing to multiple strain infections

\begin{tabular}{|c|c|c|c|c|c|c|c|c|}
\hline & \multicolumn{2}{|c|}{$2006 / 2007$} & \multicolumn{2}{|c|}{$2007 / 2008$} & \multicolumn{2}{|c|}{$2008 / 2009$} & \multicolumn{2}{|c|}{ Total } \\
\hline & No. & $\%$ & No. & $\%$ & No. & $\%$ & No. & $\%$ \\
\hline G12P[8] & 21 & $0 \cdot 55$ & 46 & $0 \cdot 54$ & 52 & $0 \cdot 77$ & 119 & 0.62 \\
\hline G12P[6] & 1 & $0 \cdot 03$ & 4 & $0 \cdot 05$ & 41 & $0 \cdot 61$ & 46 & $0 \cdot 24$ \\
\hline G12P-UD & 2 & $0 \cdot 05$ & 2 & $0 \cdot 02$ & 6 & $0 \cdot 09$ & 10 & $0 \cdot 05$ \\
\hline $\mathrm{G} 1+\mathrm{G} 12 \mathrm{P}[8]$ & 2 & $0 \cdot 05$ & 2 & $0 \cdot 02$ & 5 & $0 \cdot 07$ & 9 & $0 \cdot 05$ \\
\hline G12P[9] & 0 & $0 \cdot 00$ & 4 & $0 \cdot 05$ & 4 & $0 \cdot 06$ & 8 & $0 \cdot 04$ \\
\hline $\mathrm{G} 9+\mathrm{G} 12 \mathrm{P}[8]$ & 0 & $0 \cdot 00$ & 1 & $0 \cdot 01$ & 4 & $0 \cdot 06$ & 5 & $0 \cdot 03$ \\
\hline $\mathrm{G} 12 \mathrm{P}[4]$ & 0 & $0 \cdot 00$ & 0 & $0 \cdot 00$ & 3 & $0 \cdot 04$ & 3 & 0.02 \\
\hline $\mathrm{G} 4+\mathrm{G} 12 \mathrm{P}[8]$ & 0 & $0 \cdot 00$ & 2 & $0 \cdot 02$ & 1 & $0 \cdot 01$ & 3 & 0.02 \\
\hline $\mathrm{G} 3+\mathrm{G} 9+\mathrm{G} 12 \mathrm{P}[8]$ & 0 & $0 \cdot 00$ & 2 & $0 \cdot 02$ & 0 & $0 \cdot 00$ & 2 & $0 \cdot 01$ \\
\hline $\mathrm{G} 2+\mathrm{G} 12 \mathrm{P}[4]$ & 0 & $0 \cdot 00$ & 0 & $0 \cdot 00$ & 2 & $0 \cdot 03$ & 2 & $0 \cdot 01$ \\
\hline $\mathrm{G} 12 \mathrm{P}[4]+\mathrm{P}[8]$ & 0 & $0 \cdot 00$ & 1 & $0 \cdot 01$ & 1 & $0 \cdot 01$ & 2 & $0 \cdot 01$ \\
\hline $\mathrm{G} 2+\mathrm{G} 12 \mathrm{P}[4]+\mathrm{P}[8]$ & 1 & $0 \cdot 03$ & 1 & $0 \cdot 01$ & 0 & $0 \cdot 00$ & 2 & $0 \cdot 01$ \\
\hline $\mathrm{G} 8+\mathrm{G} 12 \mathrm{P}[6]+\mathrm{P}[8]$ & 0 & $0 \cdot 00$ & 0 & $0 \cdot 00$ & 2 & $0 \cdot 03$ & 2 & $0 \cdot 01$ \\
\hline $\mathrm{G} 1+\mathrm{G} 12 \mathrm{P}[6]$ & 1 & $0 \cdot 03$ & 0 & $0 \cdot 00$ & 0 & $0 \cdot 00$ & 1 & $0 \cdot 01$ \\
\hline $\mathrm{G} 1+\mathrm{G} 12 \mathrm{P}[4]$ & 0 & $0 \cdot 00$ & 0 & $0 \cdot 00$ & 1 & $0 \cdot 01$ & 1 & $0 \cdot 01$ \\
\hline $\mathrm{G} 1+\mathrm{G} 8+\mathrm{G} 12 \mathrm{P}[8]$ & 0 & $0 \cdot 00$ & 0 & $0 \cdot 00$ & 1 & $0 \cdot 01$ & 1 & $0 \cdot 01$ \\
\hline $\mathrm{G} 8+\mathrm{G} 12 \mathrm{P}[4]$ & 0 & $0 \cdot 00$ & 0 & $0 \cdot 00$ & 1 & $0 \cdot 01$ & 1 & $0 \cdot 01$ \\
\hline $\mathrm{G} 12 \mathrm{P}[8]+\mathrm{P}[9]$ & 0 & $0 \cdot 00$ & 0 & $0 \cdot 00$ & 1 & $0 \cdot 01$ & 1 & $0 \cdot 01$ \\
\hline $\mathrm{G} 12 \mathrm{P}[9]+\mathrm{P}[11]$ & 0 & $0 \cdot 00$ & 0 & $0 \cdot 00$ & 1 & $0 \cdot 01$ & 1 & $0 \cdot 01$ \\
\hline $\mathrm{G} 1+\mathrm{G} 2+\mathrm{G} 12 \mathrm{P}[4]+\mathrm{P}[8]$ & 0 & $0 \cdot 00$ & 0 & $0 \cdot 00$ & 1 & $0 \cdot 01$ & 1 & $0 \cdot 01$ \\
\hline $\mathrm{G} 2+\mathrm{G} 12 \mathrm{P}[6]+\mathrm{P}[8]$ & 0 & $0 \cdot 00$ & 0 & $0 \cdot 00$ & 1 & $0 \cdot 01$ & 1 & $0 \cdot 01$ \\
\hline $\mathrm{G} 4+\mathrm{G} 12 \mathrm{P}[4]+\mathrm{P}[8]$ & 0 & $0 \cdot 00$ & 0 & $0 \cdot 00$ & 1 & $0 \cdot 01$ & 1 & $0 \cdot 01$ \\
\hline $\mathrm{G} 9+\mathrm{G} 12 \mathrm{P}[4]+\mathrm{P}[8]$ & 0 & $0 \cdot 00$ & 1 & $0 \cdot 01$ & 0 & $0 \cdot 00$ & 1 & $0 \cdot 01$ \\
\hline $\mathrm{G} 8+\mathrm{G} 12 \mathrm{P}[4]+\mathrm{P}[6]$ & 0 & $0 \cdot 00$ & 0 & $0 \cdot 00$ & 1 & $0 \cdot 01$ & 1 & $0 \cdot 01$ \\
\hline $\mathrm{G} 9+\mathrm{G} 12 \mathrm{P}[8]+\mathrm{P}[9]$ & 1 & $0 \cdot 03$ & 0 & $0 \cdot 00$ & 0 & $0 \cdot 00$ & 1 & $0 \cdot 01$ \\
\hline $\mathrm{G} 10+\mathrm{G} 12 \mathrm{P}[6]$ & 0 & $0 \cdot 00$ & 0 & $0 \cdot 00$ & 1 & $0 \cdot 01$ & 1 & $0 \cdot 01$ \\
\hline Total G12 & 29 & $0 \cdot 76$ & 66 & $0 \cdot 77$ & 132 & 1.95 & 227 & $1 \cdot 19$ \\
\hline G8P[4] & 0 & $0 \cdot 00$ & 3 & $0 \cdot 04$ & 63 & 0.93 & 66 & $0 \cdot 34$ \\
\hline G8P[8] & 2 & $0 \cdot 05$ & 2 & $0 \cdot 02$ & 6 & $0 \cdot 09$ & 10 & $0 \cdot 05$ \\
\hline G8P[6] & 4 & $0 \cdot 11$ & 1 & $0 \cdot 01$ & 1 & $0 \cdot 01$ & 6 & 0.03 \\
\hline G8P[14] & 0 & $0 \cdot 00$ & 1 & $0 \cdot 01$ & 2 & $0 \cdot 01$ & 3 & $0 \cdot 01$ \\
\hline G3 + G8P[8] & 0 & $0 \cdot 00$ & 0 & $0 \cdot 00$ & 6 & $0 \cdot 09$ & 6 & 0.03 \\
\hline G4 + G8P[4] & 0 & $0 \cdot 00$ & 0 & $0 \cdot 00$ & 2 & $0 \cdot 03$ & 2 & $0 \cdot 01$ \\
\hline $\mathrm{G} 1+\mathrm{G} 8+\mathrm{G} 12 \mathrm{P}[8]$ & 0 & $0 \cdot 00$ & 0 & $0 \cdot 00$ & 1 & $0 \cdot 01$ & 1 & $0 \cdot 01$ \\
\hline G1 + G8P[4] & 0 & $0 \cdot 00$ & 0 & $0 \cdot 00$ & 1 & $0 \cdot 01$ & 1 & $0 \cdot 01$ \\
\hline G1 + G8P[8] & 0 & $0 \cdot 00$ & 0 & $0 \cdot 00$ & 1 & $0 \cdot 01$ & 1 & $0 \cdot 01$ \\
\hline G3 + G8P[8] & 0 & $0 \cdot 00$ & 1 & $0 \cdot 01$ & 0 & $0 \cdot 00$ & 1 & $0 \cdot 01$ \\
\hline $\mathrm{G} 8+\mathrm{G} 12 \mathrm{P}[4]$ & 0 & $0 \cdot 00$ & 0 & $0 \cdot 00$ & 1 & $0 \cdot 01$ & 1 & $0 \cdot 01$ \\
\hline $\mathrm{G} 8 \mathrm{P}[4]+\mathrm{P}[6]$ & 0 & $0 \cdot 00$ & 0 & $0 \cdot 00$ & 5 & $0 \cdot 07$ & 5 & $0 \cdot 03$ \\
\hline $\mathrm{G} 8 \mathrm{P}[6]+\mathrm{P}[8]$ & 0 & $0 \cdot 00$ & 0 & $0 \cdot 00$ & 2 & $0 \cdot 03$ & 2 & $0 \cdot 01$ \\
\hline $\mathrm{G} 8 \mathrm{P}[4]+\mathrm{P}[8]$ & 0 & $0 \cdot 00$ & 0 & $0 \cdot 00$ & 1 & $0 \cdot 01$ & 1 & $0 \cdot 01$ \\
\hline $\mathrm{G} 8+\mathrm{G} 12 \mathrm{P}[6]+\mathrm{P}[8]$ & 0 & $0 \cdot 00$ & 0 & $0 \cdot 00$ & 2 & $0 \cdot 03$ & 2 & $0 \cdot 01$ \\
\hline $\mathrm{G} 8+\mathrm{G} 12 \mathrm{P}[4]+\mathrm{P}[6]$ & 0 & $0 \cdot 00$ & 0 & $0 \cdot 00$ & 1 & $0 \cdot 01$ & 1 & $0 \cdot 01$ \\
\hline G8P-UD & 0 & $0 \cdot 00$ & 1 & $0 \cdot 01$ & 1 & $0 \cdot 01$ & 2 & $0 \cdot 01$ \\
\hline Total G8 & 6 & $0 \cdot 16$ & 9 & $0 \cdot 11$ & 95 & $1 \cdot 40$ & 110 & $0 \cdot 57$ \\
\hline
\end{tabular}

\section{Distribution according to rural or urban setting}

For those patients for whom the setting was provided, $89 \cdot 7 \%$ samples were collected from urban populations and $10 \cdot 2 \%$ from rural populations.
The percentages of the European population living in urban and rural areas are estimated to be $73 \cdot 1 \%$ and $26 \cdot 9 \%$, respectively (www.cap-lmu.de/fgz/ statistics/urban_pop.php). G1P[8] strains $\left(\chi^{2}=19 \cdot 88\right.$, $P<0.0001)$ were found significantly more often in 

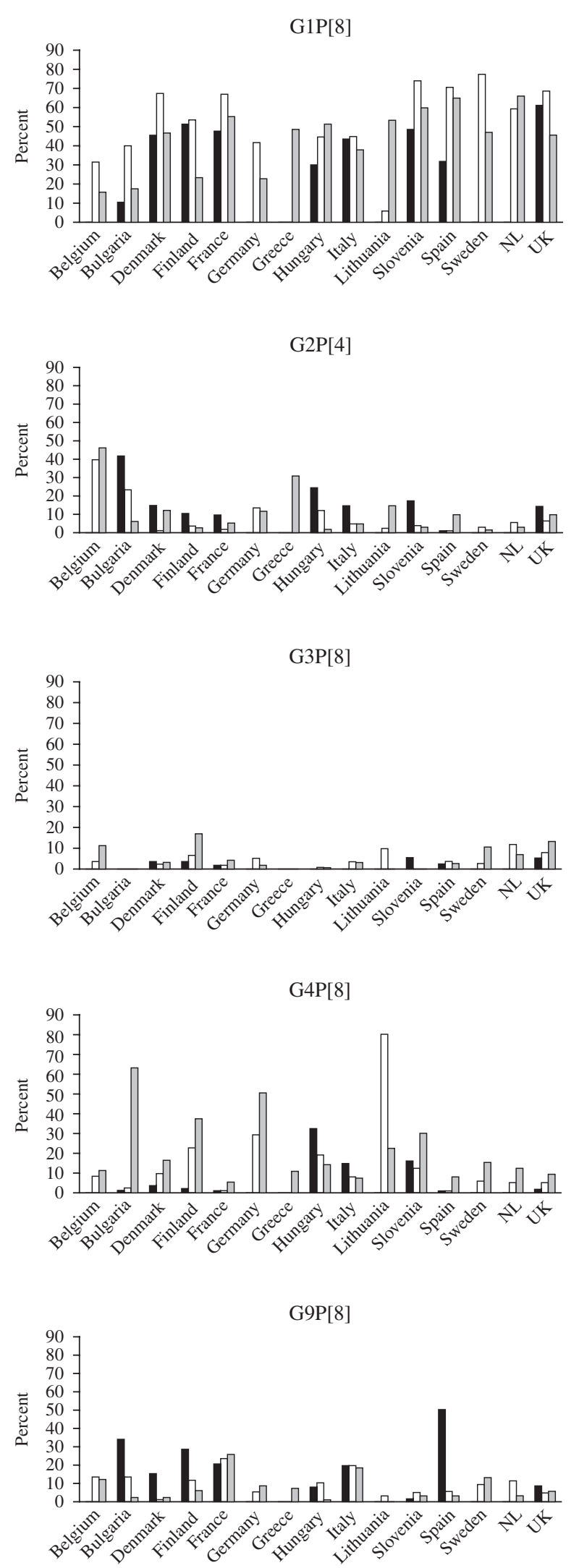

Fig. 3. Changing strain distribution in seasons and countries between 2006 and 2009. $\square, 2006 / 2007 ; \square, 2007 / 2008$; $\square$, 2008/2009. rural populations, whereas $\mathrm{G} 2 \mathrm{P}[4]\left(\chi^{2}=8 \cdot 07, P<\right.$ $0 \cdot 005)$ and G9P $[8]\left(\chi^{2}=19 \cdot 62, P<0 \cdot 0001\right)$ were found more commonly in urban populations (Table $6 b$ ). The increased incidence of G9P[8] and G2P[4] strains in the urban population may reflect the recent introduction of either an antibody escape mutant, such as seen previously with G2P[4], or an animal human reassortant, such as G9P[8]. The decreased incidence of G1P[8] in the urban population may be a result of the increased activity of G2P[4] and G9P[8] resulting in fewer susceptible individuals.

\section{Distribution according to gender}

For those subjects for whom gender was provided, $54.7 \%$ were male and $45.3 \%$ were female, which reflects the European birth cohort male:female ratio of $1 \cdot 21: 1$. No significant differences in the distribution of common or emerging rotavirus strains were found between males and females (Table $6 c$ ).

\section{DISCUSSION}

EuroRotaNet, was established in order to determine the diversity of co-circulating rotavirus strains in Europe over three or more rotavirus seasons from 2006/2007. Initially, 11 countries participated in the network and this had expanded to 16 countries by the 2008/2009 season. For analytical purposes, limited epidemiological data including age, sex, symptoms, date of onset, date of sample collection, country, region and settings, including hospital or community and rural or urban were collected.

The data on seasonality confirm the previously reported trend that rotavirus infections spread in Europe from South to North and West to East. This is similar to that observed in North America [20].

It might be expected that genotypes with a relatively low incidence are at a disadvantage in terms of transmission opportunities, and this is likely to be reflected by an increase in infections in older children. Data from Table 7, with the exception of G2P[4], do not support this, and the rationale for common strains such as G1P[8] and uncommon strains such as G8P[4] to significantly infect children between ages 14 and 15 months, is unexplained. The significant peak of infection in older children associated with G2P[4] may reflect poor cross-protection generated through previous infections with other common human rotavirus strains. Interestingly, this may reflect a replicative disadvantage of this strain in a mixture of 

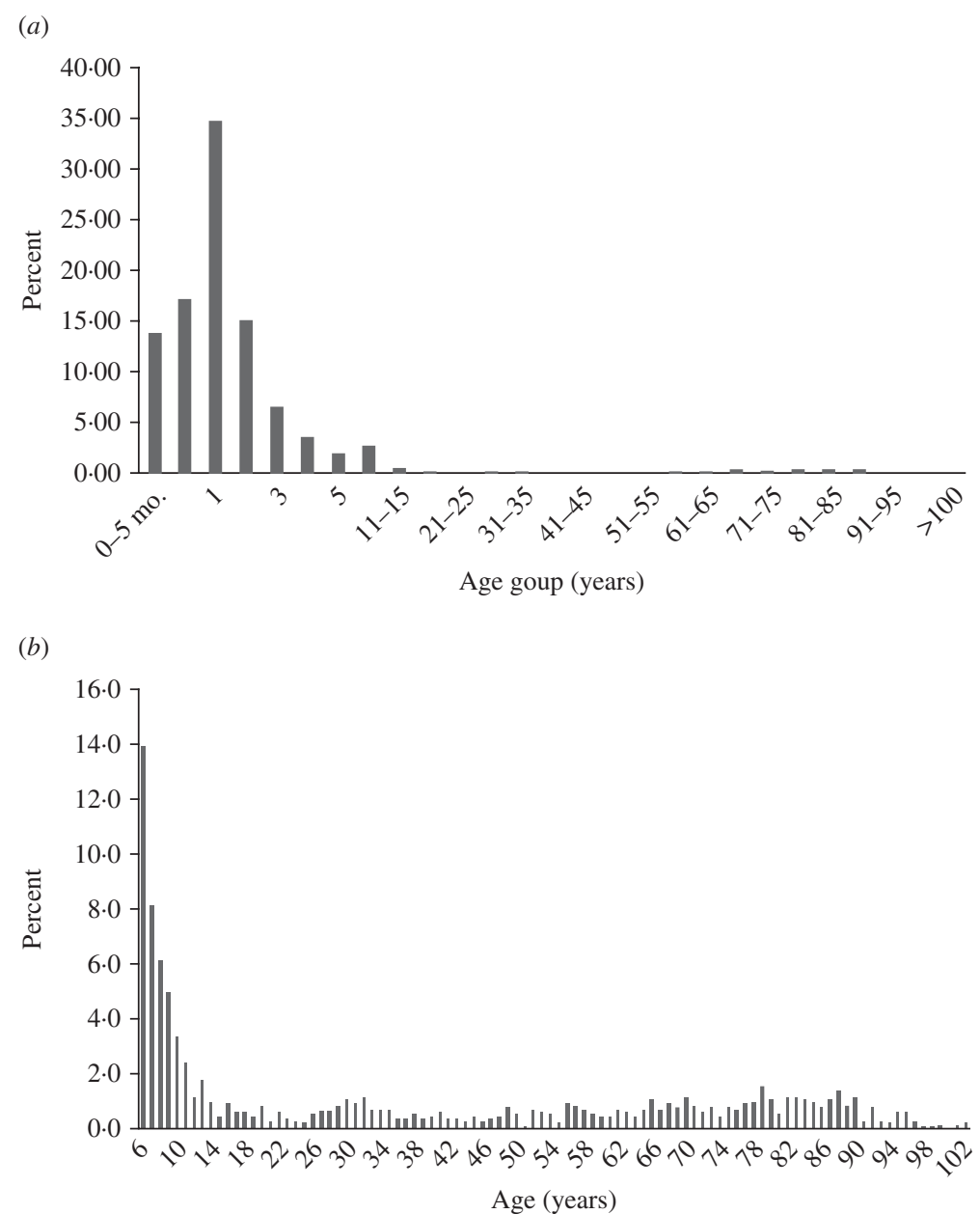

Fig. 4. Age distribution. (a) Rotavirus infections by age group in all countries $(n=17510)$. (b) Rotavirus infections from ages 6 to $>100$ years $(n=1283)$.

predominantly fit viruses infecting an immunologically naive child. This taken with the development of immunological protection against the commonly circulating strains at a young age increases the probability that any new infection in an older child is likely to be with a less common, possibly less fit strain, such as $\mathrm{G} 2 \mathrm{P}[4]$.

This report highlights the tremendous diversity of rotavirus strains co-circulating in the European population and points to the many origins of these strains including genetic reassortment and interspecies transmission. Mixed infections and zoonotic introductions are common, but many will lead to evolutionary dead-ends with only those virus strains demonstrating fitness for transmission within the human population emerging to become significant human pathogens.

The G- or P-types associated with partially typed strains were representative of those genotypes seen in fully characterized strains. Therefore, failure to type is most likely to be associated with technical issues and/or low viral load, although occasionally they may represent usual genotypes, particularly in those associated with an animal rotavirus G- or P- types [21-26]. The failure to identify G-types associated with $\mathrm{P}[6], \mathrm{P}[9], \mathrm{P}[10], \mathrm{P}[11]$ and $\mathrm{P}[14]$ may be associated with the presence of unusual G-types for which G genotype-specific primers were not included in the study protocol. Similarly, the inability to determine the P-type of strains with G12 or G10 may be due to a lack of $\mathrm{P}$ genotype-specific primers. Interestingly, other strains associated with these G- or P-types were fully characterized during the study.

With the ability of the network to identify strains circulating with an incidence of $1 \%$, possible emerging strains such as G8 and G12 have been identified during the first 3 years of the study and the analysis of recent data indicates their increased incidence.

G12P[6] and G12P[14] are likely to represent zoonotic spread into the human population, whereas, 
Table 6. Distribution of common and emerging rotavirus genotypes. The total number of strains shown refers to the total number of rotavirus strains in each of the settings/gender groups, and includes mixed infections and human rotavirus reassortant strains (not shown in the table breakdown)

(a) Hospitalized and community cases

\begin{tabular}{|c|c|c|c|c|}
\hline \multirow[t]{2}{*}{ Genotype } & \multicolumn{2}{|c|}{ Hospital } & \multicolumn{2}{|c|}{ Community } \\
\hline & No. & $\%$ & No. & $\%$ \\
\hline G1P[8] & 5008 & $48 \cdot 24$ & 2900 & $46 \cdot 27$ \\
\hline $\mathrm{G} 2 \mathrm{P}[4]$ & 1106 & $10 \cdot 65$ & 524 & $8 \cdot 36$ \\
\hline G3P[8] & 383 & $3 \cdot 69$ & 336 & $5 \cdot 36$ \\
\hline G4P[8] & 1673 & $16 \cdot 11$ & 924 & $14 \cdot 74$ \\
\hline G9P[8] & 1228 & $11 \cdot 83$ & 801 & $12 \cdot 78$ \\
\hline G12 with any & 137 & $1 \cdot 32$ & 77 & $1 \cdot 23$ \\
\hline G8 with any & 66 & 0.64 & 42 & 0.67 \\
\hline Total typed & 10382 & & 6267 & \\
\hline
\end{tabular}

(b) Urban and rural settings

\begin{tabular}{|c|c|c|c|c|}
\hline \multirow[t]{2}{*}{$\overline{\overline{\text { Genotype }}}$} & \multicolumn{2}{|l|}{ Urban } & \multicolumn{2}{|c|}{ 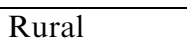 } \\
\hline & No. & $\%$ & No. & $\%$ \\
\hline G1P[8] & 2677 & $39 \cdot 13$ & 371 & $47 \cdot 44$ \\
\hline G2P[4] & 976 & $14 \cdot 26$ & 82 & $10 \cdot 49$ \\
\hline G3P[8] & 231 & $3 \cdot 38$ & 29 & $3 \cdot 71$ \\
\hline G4P[8] & 1258 & $18 \cdot 39$ & 168 & $21 \cdot 48$ \\
\hline G9P[8] & 792 & $11 \cdot 58$ & 49 & $6 \cdot 27$ \\
\hline G12 with any & 101 & $1 \cdot 48$ & 11 & $1 \cdot 41$ \\
\hline G8 with any & 24 & $0 \cdot 35$ & 5 & $0 \cdot 64$ \\
\hline Total typed & 6842 & & 782 & \\
\hline
\end{tabular}

(c) Males and females

\begin{tabular}{|c|c|c|c|c|}
\hline \multirow[t]{2}{*}{ Genotype } & \multicolumn{2}{|l|}{ Male } & \multicolumn{2}{|c|}{ Female } \\
\hline & No. & $\%$ & No. & $\%$ \\
\hline G1P[8] & 4529 & $47 \cdot 63$ & 3710 & $47 \cdot 17$ \\
\hline G2P [4] & 953 & $10 \cdot 02$ & 809 & $10 \cdot 29$ \\
\hline G3P[8] & 412 & $4 \cdot 33$ & 336 & $4 \cdot 27$ \\
\hline G4P[8] & 1473 & $15 \cdot 49$ & 1286 & $16 \cdot 35$ \\
\hline G9P[8] & 1142 & $12 \cdot 01$ & 933 & $11 \cdot 86$ \\
\hline G12 with any & 95 & $1 \cdot 00$ & 107 & $1 \cdot 36$ \\
\hline G8 with any & 56 & $0 \cdot 59$ & 44 & $0 \cdot 56$ \\
\hline Total typed & 9508 & & 7865 & \\
\hline
\end{tabular}

G12P[4] and G12P[8] are more likely to be the result of reassortment in human and animal strains. The plethora of mixtures of rotaviruses containing G12 strains may suggest environmental or food-/waterborne transmission events. Mixtures can also be seen to contain potential zoonotic strains other than
Table 7. Median age of infection compared with overall incidence by genotype

\begin{tabular}{llr}
\hline \hline & $\begin{array}{l}\text { Median age } \\
\text { of infections } \\
\text { (months) }\end{array}$ & Incidence (\%) \\
Genotype & 15 & $48 \cdot 43$ \\
G1P[8] & 22 & $10 \cdot 07$ \\
G2P[4] & 15 & $4 \cdot 27$ \\
G3P[8] & 18 & $15 \cdot 06$ \\
G4P[8] & 15 & $11 \cdot 56$ \\
G9P[8] & 14 & $0 \cdot 34$ \\
G8P[4] & 19 & $1 \cdot 19$ \\
G12 with any P & 18 & $4 \cdot 22$ \\
Mixed genotypes & & \\
\hline \hline
\end{tabular}

G12 such as G8, G10, P[6], P[9] and P[11] and so may represent an environmental reservoir of rotavirus strains associated with a mixture of potentially contaminating human and animal faeces. Similarly, G8P[14] and G8P[6] may be zoonotic, whereas, G8P[4] and G8P[8] may result from reassortment of an animal strain carrying G8, with human strains. Twenty-four mixtures made up of 12 different combinations of G- and/or P-types were found, suggesting infection in some instances through contact with a mixture of human and animal strains.

The genes encoding VP7 of the G8 strains from the UK were sequenced. The temporal and geographical distribution and diversity of G8 strains seen before 2009 may suggest multiple zoonotic introductions $[13,27-33]$ whereas the similarity of the strains described in the current paper is likely to be the result of sustained person-to-person transmission of a strain adapted to the human host (Fig. 5). Interestingly, when G12 strains from UK, Hungary and Bulgaria were sequenced, detailed phylogenetic analysis indicated multiple, unrelated, introductions and may represent a series of evolutionary dead-ends associated with an inability of these strains to sustain human-to-human transmission (Fig. 6). It is reasonable to suggest that because of the frequent introductions of G12 strains into the human population it may be only a matter of time before a reassortment event provides a G12 animal/human hybrid strain of comparable fitness to those current stains circulating commonly in the human population.

The introduction of universal rotavirus vaccination in at least two of the participating countries, and partial vaccine coverage in some others may provide data on diversity driven by vaccine introduction and possible strain replacement. Discussions on changes to the current EuroRotaNet surveillance protocols in 
VP7

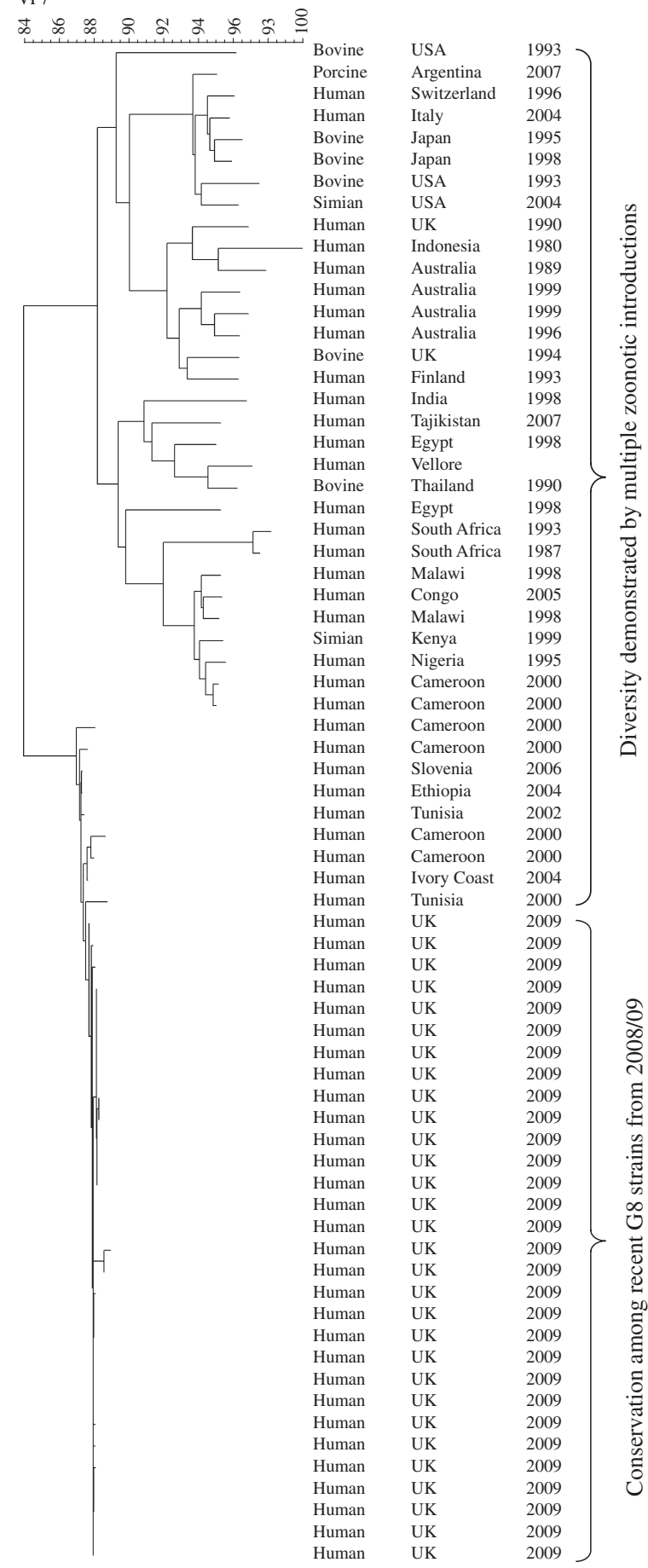

Fig. 5. Dendrogram (Neighbour Joining) of sequences of the gene encoding VP7 of G8 strains. Host, country and year of identification are indicated after each strain.

light of rotavirus vaccine introduction will be held during the course of the next two seasons and will involve participants, funders and the European Centre for Diseases Control (ECDC). In light of these developments, it should now be possible for

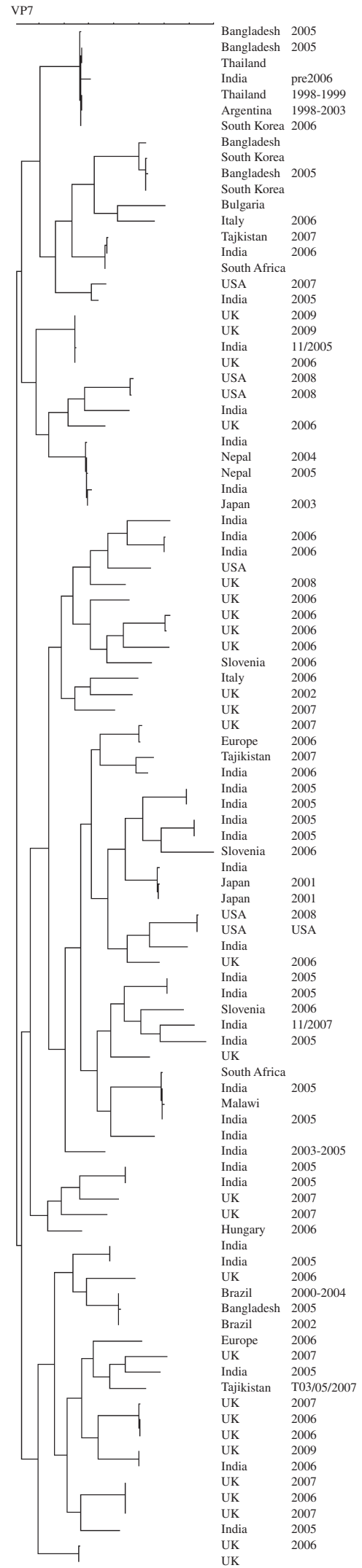

Fig. 6. Dendrogram (Maximum Parsimony) of sequences of the gene encoding VP7 of G12 strains. Strains included are global and represent introductions between 1991 and 2009. 
EuroRotaNet, with others, to evolve into a network capable of performing detailed rotavirus surveillance activities. These activities would provide data on the effectiveness of rotavirus vaccination programmes and the impact of these programmes on the virus populations co-circulating in Europe, and the changing patterns of infections by age, season, setting, etc.

\section{DECLARATION OF INTEREST}

EuroRotaNet receives funding from GSK and SPMSD in equal parts through a non-restrictive collaborative grant.

\section{REFERENCES}

1. Parashar UD, et al. Rotavirus and severe childhood diarrhea. Emerging Infectious Diseases 2006; 12: 304 306.

2. Parashar UD, et al. Global illness and deaths caused by rotavirus disease in children. Emerging Infectious Diseases 2003; 9: 565-572.

3. Greenberg HB, et al. Serological analysis of the subgroup protein of rotavirus, using monoclonal antibodies. Infection and Immunity 1983; 39: 91-99.

4. Iturriza Gomara M, et al. Molecular characterization of VP6 genes of human rotavirus isolates: correlation of genogroups with subgroups and evidence of independent segregation. Journal of Virology 2002; 76: 6596-6601.

5. Abe M, et al. Molecular epidemiology of rotaviruses among healthy calves in Japan: isolation of a novel bovine rotavirus bearing new $\mathrm{P}$ and $\mathrm{G}$ genotypes. Virus Research 2009; 144: 250-257.

6. Matthijnssens $\mathbf{J}$, et al. Recommendations for the classification of group A rotaviruses using all 11 genomic RNA segments. Archives of Virology 2008; 153: 1621-1629.

7. Schumann T, et al. Evidence of interspecies transmission and reassortment among avian group A rotaviruses. Virology 2009; 386: 334-343.

8. Solberg OD, et al. Characterization of novel VP7, VP4, and VP6 genotypes of a previously untypeable group A rotavirus. Virology 2009; 385: 58-67.

9. Ursu K, et al. Molecular analysis of the VP7 gene of pheasant rotaviruses identifies a new genotype, designated G23. Archives of Virology 2009; 154: 1365-1369.

10. Garbarg-Chenon A, Bricout F, Nicolas J. Study of genetic reassortment between two human rotaviruses. Virology 1984; 139: 358-365.

11. Gombold JL, Ramig RF. Analysis of reassortment of genome segments in mice mixedly infected with rotaviruses SA11 and RRV. Journal of Virology 1986; 57 : $110-116$.

12. Unicomb LE, et al. Evidence of high-frequency genomic reassortment of group A rotavirus strains in
Bangladesh: emergence of type G9 in 1995. Jounal of Clinical Microbiology 1999; 37: 1885-1891.

13. Esona MD, et al. Genomic characterization of human rotavirus G8 strains from the African rotavirus network: relationship to animal rotaviruses. Journal of Medical Virology 2009; 81: 937-951.

14. Iturriza-Gomara M, Desselberger U, Gray J. Molecular epidemiology of rotaviruses: genetic mechanisms associated with diversity. In: Desselberger U, Gray J, eds. Viral Gastroenteritis. Amsterdam: Elsevier, 2003, pp. 317-344.

15. McDonald SM, et al. Evolutionary dynamics of human rotaviruses: balancing reassortment with preferred genome constellations. PLoS Pathogogy 2009; 5: e1000634.

16. Matthijnssens $\mathbf{J}$, et al. Simian rotaviruses possess divergent gene constellations that originated from interspecies transmission and reassortment. Journal of Virology 2010; 84: 2013-2026.

17. Iturriza-Gomara $\mathbf{M}$, et al. Rotavirus surveillance in europe, 2005-2008: web-enabled reporting and realtime analysis of genotyping and epidemiological data. Journal of Infectious Diseases 2009; 200 (Suppl. 1): S215-221.

18. Iturriza-Gomara M, et al. Evidence for genetic linkage between the gene segments encoding NSP4 and VP6 proteins in common and reassortant human rotavirus strains. Journal of Clinical Microbiology 2003; 41: 3566-3573.

19. Iturriza-Gómara M, et al. Reassortment in vivo: driving force for diversity of human rotavirus strains isolated in the United Kingdom between 1995 and 1999. Journal of Virology 2001; 75: 3696-3705.

20. Turcios RM, et al. Temporal and geographic trends of rotavirus activity in the United States, 1997-2004. Pediatric Infectious Diseases Journal 2006; 25 : 451-454.

21. Iturriza Gomara M, et al. Characterization of G10P[11] rotaviruses causing acute gastroenteritis in neonates and infants in Vellore, India. Journal of Clinical Microbiology 2004; 42 : 2541-2547.

22. Iturriza-Gómara M, et al. Diversity within the VP4 gene of rotavirus $\mathrm{P}[8]$ strains: implications for reverse transcription-PCR genotyping. Journal of Clinical Microbiology 2000; 38: 898-901.

23. Iturriza-Gomara M, Kang G, Gray JJ. Rotavirus genotyping: keeping up with an evolving population of human rotaviruses. Journal of Medical Virology 2004; 31: 259-265.

24. Simmonds MK, et al. New oligonucleotide primers for P-typing of rotavirus strains: Strategies for typing previously untypeable strains. Journal of Clinical Virology 2008; 42: 368-373.

25. Adah MI RA, Olaleyle, Werchau H. Nigerian rotavirus serotypoe G8 could not be typed by PCR due to nucleotide mutation at the $3^{\prime}$ end of the primer site. Archives of Virology 1997; 142: 1881-1887.

26. Iturriza Gómara M, et al. Amino acid substitution within the VP7 protein of G2 rotavirus strains associated with failure to serotype. Journal of Clinical Microbiology 2001; 39: 3796-3798. 
27. Alfieri AF, et al. G and $P$ genotypes of group A rotavirus strains circulating in calves in Brazil, 1996-1999. Veterinary Microbiology 2004; 99: 167-173.

28. Browning GF, et al. Human and bovine serotype G8 rotaviruses may be derived by reassortment. Archives of Virology 1992; 125: 121-128.

29. Cunliffe NA, et al. Molecular and serologic characterization of novel serotype G8 human rotavirus strains detected in Blantyre, Malawi. Virology 2000; 274: 309-320.

30. Fischer TK, et al. Characterization of incompletely typed rotavirus strains from Guinea-Bissau: identification of G8 and G9 types and a high frequency of mixed infections. Virology 2003; 311 : 125-133.

31. Holmes JL, et al. Characterization of unusual G8 rotavirus strains isolated from Egyptian children. Epidemiology and Infection 1999; 123: 9-16.

32. Salu OB, et al. Molecular epidemiology of rotaviruses in Nigeria: detection of unusual strains with G2P[6] and G8P[1] specificities. Journal of Clinical Microbiology $2003 ; 41$ : 913-914.

33. Santos N, et al. Detection of rotavirus types G8 and G10 among Brazilian children with diarrhea. Journal of Clinical Microbiology 1998; 36: 2727-2729. 